九州大学学術情報リポジトリ

Kyushu University Institutional Repository

Energy of hydrodynamic and magnetohydrodynamic waves with point and continuous spectra

Hirota, Makoto

Faculty of Mathematics, Kyushu University

Fukumoto, Yasuhide

Faculty of Mathematics, Kyushu University

http://hdl. handle. net/2324/8709

出版情報 : MHF Preprint Series. 2007-17，2007-12-12. 九州大学大学院数理学研究院 バージョン：

権利関係 : 


\section{MHF Preprint Series}

Kyushu University

21st Century COE Program

Development of Dynamic Mathematics with High Functionality

Energy of hydrodynamic and magnetohydrodynamic waves with point and continuous spectra

\section{Hirota \& Y. Fukumoto}

MHF 2007-17

( Received December 12, 2007 )

Faculty of Mathematics

Kyushu University

Fukuoka, JAPAN 


\title{
Energy of hydrodynamic and magnetohydrodynamic waves with point and continuous spectra
}

\author{
M. Hirota and Y. Fukumoto \\ Faculty of Mathematics, Kyushu University, Fukuoka, 812-8581, Japan

\begin{abstract}
Energy of waves (or eigenmodes) in ideal fluid and plasma is formulated in the noncanonical Hamiltonian context. By imposing the kinematical constraint on perturbations, the linearized Hamiltonian equation provides a formal definition of wave energy not only for eigenmodes corresponding to point spectra but also for singular ones corresponding to continuous spectrum. The latter becomes dominant when mean fields have inhomogeneity originating from shear or gradient of the fields. The energy of each wave is represented by the eigenfrequency multiplied by the wave action which is nothing but the action variable and, moreover, is associated with a derivative of suitably defined dispersion relation. The sign of the action variable is crucial to the occurrence of Hopf bifurcation in Hamiltonian systems of a finite degrees of freedom (Krein 1950). Krein's idea is extended to the case of coalescence
\end{abstract} \\ between point and continuous spectra.
}

\section{INTRODUCTION}

Evaluating the energy contained in the hydrodynamic (HD) and magnetohydrodynamic (MHD) waves is important especially in the context of stability analysis and bifurcation theory. If the energy of any perturbation away from an equilibrium state is positive (or negative) definite, such a state turns out to be nonlinearly stable [1-3], although it gives rather strong sufficient condition for stability. More detailed stability condition is investigated by solving the linearized dynamical system. The linear waves and their frequencies correspond respectively to the eigenmodes and the eigenvalues of the linear system, some of which may have negative energy if the corresponding perturbation extracts energy from the equilibrium state (i.e., the mean fields). The negative energy mode is in itself neutrally stable (unless the system undergoes any dissipation effect).

According to the general theory for Hamiltonian systems of finite dimension, it was shown by Krein [4] and Moser [5] that linear instability is only possible when a pair of eigenvalues of positive and negative energy modes or of zero energy modes collide. A deeper insight into this process, so-called Krein collision or (Hamiltonian) Hopf bifurcation, can be obtained by carrying out some canonical transformation to the action-angle variables $[5,6]$, where the energy of a single harmonic oscillator will be represented by

$$
(\text { energy })=(\text { frequency }) \times(\text { action }) \text {. }
$$

The action variable is known to be an adiabatic invariant [1], which is robust even when the equilibrium state experiences a sufficiently slow variation. Consequently, the signature of the action variable is more closely related to the bifurcation process than that of the energy.

Is is well-known that ideal fluids and plasmas are (noncanonical) Hamiltonian systems. Hence the expression of energy for HD and MHD waves is expected to have similarity with (1). Historically, the expression for wave energy was first gained in early works on 
electromagnetic waves in homogeneous plasmas $[7,8]$; the energy of a plane wave with a frequency $\omega$ and a wave number $k$, satisfying the dispersion relation $D(\omega, k)=0$, is given by

$$
\text { (wave energy) }=\omega \frac{\partial D}{\partial \omega}(\omega, k)|\boldsymbol{E}(\omega, k)|^{2},
$$

where $\boldsymbol{E}(\omega, k)$ is the amplitude of the perturbed electric field. Since the Hamiltonian structure was not recognized at that time, the above expression was derived from estimation of the amount of work done by the source current which slowly excites this wave. The wave action is obviously $\partial D / \partial \omega|\boldsymbol{E}|^{2}$, whose sign is determined by the $\omega$-derivative of the dispersion relation. This expression carries over to inhomogeneous plasmas in the shortwavelength limit $[9,10]$, which has been widely used in the wave theory and nowadays called the Wentzel-Kramers-Brillouin (WKB) approximation.

The same argument is immediately applied to the energy of electrostatic waves in hot plasmas. More recently, Morrison and Pfirsch [11] compared this expression (2) with the exact plasma free energy by invoking the Hamiltonian structure of the Vlasov-Poisson equation. It is remarkable that they also derived an expression for wave energy associated with the continuous spectrum. Their result indicates that the formula (2) is true for point spectra (eigenvalues), but not for continuous spectrum. The continuous spectrum is yet to be explored in the Hamiltonian mechanics because it never occurs in finite degree-of-freedom systems. Since the corresponding waves are represented by singular eigenfunctions so-called the Van Kampen modes [12], the extensive use of the singular integral transformation (like the Hilbert transform) is required to attain the action-angle representation.

Although the electric field never intervenes in fluid mechanics, Cairns [13] pointed out the existence of a similar expression to (2) for waves in parallel flows with piecewise-constant velocity and density profiles. The wave energy was estimated from the amount of work required to displace the vortex sheet at the discontinuity so that the wave would be excited, which indeed results in (2) with $\boldsymbol{E}$ being replaced by the amplitude of the displacement. Thus, the Kelvin-Helmholtz instability was understood as a Hopf bifurcation. MacKay and Saffman [14] have considered the energy of water waves in the same manner. Application of the above energy criterion remains valid for arbitrary piecewise-constant mean fields.

However, there have not been so many pieces of works that discuss the HD and MHD wave energy for more general mean fields (without relying on the WKB approximation), which is the central motivation of this work. We remark that this problem is complicated by the following three points. First, if the mean fields have smooth inhomogeneity (shear or gradient), the continuous spectrum occurs in ideal HD and MHD waves, which occupies a certain region on the real axis of the complex frequency $(\omega)$ plane. The treatment of singular eigenfunctions is then nontrivial as is the case for the Van Kampen modes. A paper written by Balmforth and Morrison [15] seems to be the only work that discusses the wave energy for continuous spectrum in smooth shear flow, although their analysis was focused on incompressible and purely two-dimensional motions. Second, the dispersion relation for point spectra must be no longer an algebraic relation between $\omega$ and $k$ but a differential equation since the inhomogeneity of the mean fields does not allow the use of wavenumber $k$. Third, as was noted in Ref. 11 and 15, a kinematical constraint (so-called dynamical accessibility) must be imposed on perturbations because the Hamiltonian system is noncanonical. This constraint is already prescribed in the noncanonical Poisson bracket and is, in physical terms, characterized by the kinematical conservation laws. 
In this paper, we will formulate wave energy (as well as wave action) in a general manner based on the noncanonical Hamiltonian context. The governing equations for ideal fluid and plasma in the Eulerian description are known to have a common Hamiltonian structure socalled the Lie-Poisson bracket $[1,16-18]$. In Sec. II, we will review the general Lie-Poisson system and discuss its linearized structure about arbitrary steady state. In general, any Hamiltonian flow in the phase space must be restricted to the symplectic leaves for the Lie-Poisson bracket, which corresponds to the kinematical constraint [3]. The kinematically accessible perturbations are then identified as the tangent space to the symplectic leaves. It is reviewed by Arnold and Khesin [2] that a steady state is characterized as an extremum point of the Hamiltonian within a symplectic leaf, and the second variation of the Hamiltonian along the same symplectic leaf is the first integral of the linearized equation, by which we can define the energy of perturbation. All perturbations in this work are assumed to be kinematically accessible from the initial state. While this assumption does not perfectly cover all perturbations, it has been invoked in many physical applications, and most HD and MHD waves of interest are kinematically accessible.

In Sec. III, we will decompose the linear perturbation into waves (= eigenmodes) by means of the spectral analysis, and formulate the wave energy for both point and continuous spectra. In this work, we employ the Laplace transform approach to deal with the spectral problem. We establish a generalized dispersion relation for inhomogeneous mean fields in such a way as to be associated with the wave action.

In Sec. IV, the MHD case will be discussed. The linearized MHD equation together with the kinematically accessible initial data will be reduced to the well-known equation derived by Frieman and Rotenberg [19]. It is an evolution equation for the Lagrangian displacement field that has been widely used for the MHD stability analysis. The energy of perturbation given in Ref. 19 agrees with the second variation of the Hamiltonian [20]. New generalized dispersion relation can be defined for this Frieman and Rotenberg equation. In terms of it, we will show that a formula similar to (2) holds for any (semi-simple) point spectrum, whereas this formula does not seem to be valid for a continuous spectrum. The elimination of the variables is fundamental not only for simplifying the equation but also for obtaining simpler dispersion relation. If the mean fields were homogeneous, our generalized dispersion relation would be ultimately reduced to the conventional algebraic relation between $\omega$ and $k$ with the use of the Fourier transform in space. On the other hand, for mean fields with one-dimensional inhomogeneity, the equation is reduced to the eigenvalue problem of the Sturm-Liouville type $[21,22]$. It will be shown that the corresponding dispersion relation is again related to the wave action.

In Sec. $\mathrm{V}$, we will study more about the wave energy for a continuous spectrum and its role in the bifurcation theory. By neglecting the magnetic field for simplicity, we will concentrate on the continuous spectrum in parallel shear flows. An expression for the wave energy, which includes the result of Balmforth and Morrison [15], will be derived by exploiting some techniques, where a suitable change of variable is required to treat the singularity of the eigenfunction. This result can explain, by using the signs of wave actions, the mechanism for both destabilization [23, 24] and the Landau damping [25] of neutral waves in shear flow, which is generally understood as a Hopf bifurcation caused by collision between point and continuous spectra. 


\section{LINEARIZED LIE-POISSON SYSTEMS}

Theories for dynamical systems have been developed in mathematics so that fluids and plasmas can be dealt within the both Hamiltonian and Lagrangian formalisms. The notions of the conservation law and the topological invariant become more sophisticated with the help of the differential geometry and the Lie group theory. The noncanonical Hamiltonian structure of the MHD equation was uncovered by Morrison and Greene [26]. It was also derived by the Lie-Poisson reduction from a canonical system for the Lagrangian variables $[16,17]$. The MHD equation is, thus, regarded as a Lie-Poisson system on the dual $\mathfrak{g}^{*}$ of the Lie algebra, $\mathfrak{g}=\mathfrak{X}(\mathrm{S})\left(\Lambda^{1} \oplus \Lambda^{0} \oplus \Lambda^{3}\right)$, where $\mathfrak{X}$ and $\Lambda^{n}$ denote, respectively, the spaces of vector fields and $n$-forms on the domain $D \subset \mathbb{R}^{3}$, and (S) denotes the semidirect product. Denote an element of the dual space by $u=(\boldsymbol{M}, \boldsymbol{B}, \rho, s)^{T} \in \mathfrak{g}^{*}$, where $u$ is composed of fluid momentum density $\boldsymbol{M}$, magnetic field $\boldsymbol{B}$, mass density $\rho$ and specific entropy $s$. The velocity field $\boldsymbol{v}$ is given by $\boldsymbol{M}=\rho \boldsymbol{v}$. For any functionals $F, G: \mathfrak{g}^{*} \rightarrow \mathbb{R}$, the Lie-Poisson bracket is generically written in the form of

$$
\{F, G\}=\left\langle u,\left[\frac{\delta F}{\delta u}, \frac{\delta G}{\delta u}\right]\right\rangle
$$

using the standard pairing $\langle\rangle:, \mathfrak{g}^{*} \times \mathfrak{g} \rightarrow \mathbb{R}$ and the Lie bracket [,] $: \mathfrak{g} \times \mathfrak{g} \rightarrow \mathfrak{g}$. The Hamiltonian equation $\partial_{t} F=\{F, H\}$ is posed for a prescribed Hamiltonian function $H$ : $\mathfrak{g}^{*} \rightarrow \mathbb{R}$. In the MHD case, it represents the total energy as usual;

$$
H(u)=\int\left[\frac{1}{2 \rho}|\boldsymbol{M}|^{2}+\frac{1}{2}|\boldsymbol{B}|^{2}+\rho e(\rho, s)\right] \mathrm{d}^{3} x,
$$

where $e(\rho, s)$ is a given function of $\rho$ and $s$ representing the internal energy per unit mass. The Lie-Poisson bracket for the MHD equation is explicitly shown in several works $[3,17$, 20, 26].

Let us introduce a notation in accordance with the adjoint representation of the Lie group theory [1]. A linear operator $\operatorname{ad}(\zeta): \mathfrak{g} \rightarrow \mathfrak{g}$ for any $\zeta \in \mathfrak{g}$ is defined by

$$
\left[\zeta_{1}, \zeta_{2}\right]=-\left[\zeta_{2}, \zeta_{1}\right]=\operatorname{ad}\left(\zeta_{1}\right) \zeta_{2}=-\operatorname{ad}\left(\zeta_{2}\right) \zeta_{1} \quad \text { for } \forall \zeta_{1}, \zeta_{2} \in \mathfrak{g}
$$

The dual operator of $\operatorname{ad}(\zeta)$ with respect to the inner bracket $\langle$,$\rangle is denoted by \operatorname{ad}^{*}(\zeta): \mathfrak{g}^{*} \rightarrow$ $\mathfrak{g}^{*}$. Using this notation, the Hamiltonian equation allows another expression;

$$
\partial_{t} u=-\operatorname{ad}^{*}\left(\frac{\delta H}{\delta u}\right) u
$$

Any Lie-Poisson system is commonly written in this form of nonlinear evolution equation. Actually, one can reproduce the ideal MHD equation by substituting the corresponding Lie-Poisson bracket and the Hamiltonian (4).

In this section, we shall prepare some general properties of the Lie-Poisson system (6) linearized about an equilibrium state. While we will be mainly concerned with the MHD case (or the HD case by dropping the magnetic field $\boldsymbol{B}$ ) later, the theories presented here are pertinent to any Lie-Poisson system.

First of all, it is important to notice that the operator $\operatorname{ad}^{*}(0)$ determines, to some extent, how $u(t)$ evolves infinitesimally at each time. Suppose that the Hamiltonian in $(6)$ is replaced 
by arbitrary linear functional, i.e., $H=\langle u, \zeta\rangle$ for some $\zeta \in \mathfrak{g}$. The corresponding virtual variation $\delta u=-\operatorname{ad}^{*}(\zeta) u$ is said to be kinematically accessible (or dynamically accessible, according to Morrison [27]). Such variations $\delta u$ generated by every $\zeta \in \mathfrak{g}$ do not span the whole space $\mathfrak{g}^{*}$, which implies that there is some constraint on the dynamics. The existence of such kinematical constraints is peculiar to the noncanonical systems and, physically, it is related to the conservation laws.

Let us refer to the MHD case as an example. By denoting the components of $\zeta$ by $\zeta=(\boldsymbol{\xi}, \boldsymbol{\eta}, \alpha, \beta)^{T} \in \mathfrak{g}$, the kinematically accessible variation, $\delta u=-\operatorname{ad}^{*}(\zeta) u$, is explicitly written as

$$
\begin{aligned}
\delta \boldsymbol{v} & =\boldsymbol{\xi} \times(\nabla \times \boldsymbol{v})-\frac{1}{\rho} \boldsymbol{B} \times(\nabla \times \boldsymbol{\eta})-\nabla(\boldsymbol{\xi} \cdot \boldsymbol{v}+\alpha)+\frac{\beta}{\rho} \nabla s, \\
\delta \boldsymbol{B} & =\nabla \times(\boldsymbol{\xi} \times \boldsymbol{B}), \\
\delta \rho & =-\nabla \cdot(\rho \boldsymbol{\xi}), \\
\delta s & =-\boldsymbol{\xi} \cdot \nabla s .
\end{aligned}
$$

One can confirm that these restricted variations automatically satisfy all kinematical conservation laws of MHD, namely, do not perturb all Casimir invariants [20]. This idea first appeared in fluid mechanics as the so-called isovortical variation [1] since the above $\delta \boldsymbol{v}$ preserves the vorticity conservation (or the Kelvin's circulation theorem) in the isentropic and hydrodynamic limit; $\nabla s=0$ and $\boldsymbol{B}=0$. The variation (7)-(10) is also called the generalized isovortical variation in this context [28, 29].

A state $u_{e} \in \mathfrak{g}^{*}$ is an equilibrium (or steady) state

$$
\operatorname{ad}^{*}\left(\left.\frac{\delta H}{\delta u}\right|_{e}\right) u_{e}=0
$$

if and only if

$$
\left\langle\operatorname{ad}^{*}(\zeta) u_{e},\left.\frac{\delta H}{\delta u}\right|_{e}\right\rangle=0 \quad \text { for all } \zeta \in \mathfrak{g}
$$

where $\delta H /\left.\delta u\right|_{e}$ denotes the value of the functional derivative $\delta H / \delta u \in \mathfrak{g}$ at $u=u_{e}$. Therefore, any equilibrium state is characterized by an extremum point $(\delta H=0)$ of $H$ with respect to the kinematically accessible variations.

The linearization of (6) about this $u_{e}$ leads to a linear evolution equation for perturbation $\tilde{u}(t) \in \mathfrak{g}^{*}$ (see App. A of Ref. 3);

$$
\partial_{t} \tilde{u}=(\mathcal{A H}+\mathcal{B}) \tilde{u}, \quad \tilde{u}(0)=\tilde{u}_{0},
$$

where we defined some linear operators, $\mathcal{A}: \mathfrak{g} \rightarrow \mathfrak{g}^{*}, \mathcal{H}: \mathfrak{g}^{*} \rightarrow \mathfrak{g}$ and $\mathcal{B}: \mathfrak{g}^{*} \rightarrow \mathfrak{g}^{*}$ by

$$
\mathcal{A}:=-\operatorname{ad}^{*}(\circ) u_{e}, \quad \mathcal{H}:=\left.\frac{\delta^{2} H}{\delta u^{2}}\right|_{e} \text { and } \mathcal{B}:=-\operatorname{ad}^{*}\left(\left.\frac{\delta H}{\delta u}\right|_{e}\right) \text {. }
$$

Note that $\mathcal{A}$ is an anti-symmetric operator, $\mathcal{A}^{*}=-\mathcal{A}$ (due to the anti-symmetry of the Lie bracket), and $\mathcal{H}$ is a symmetric operator, $\mathcal{H}^{*}=\mathcal{H}$, with respect to the pairing $\langle$,$\rangle . Since$ this paper will focus on the linear theory, we will omit the subscript $e$ in what follows and $u$ will always refer to the equilibrium state satisfying (11). One can check that the linearized 
MHD equation is written in this form, where the operators $\mathcal{A}, \mathcal{H}$ and $\mathcal{B}$ are explicitly listed in Appendix A.

We consider the kinematically accessible perturbations $\tilde{u} \in\{\mathcal{A} \zeta ; \zeta \in \mathfrak{g}\}$ to the equilibrium state, which corresponds to the range of $\mathcal{A}$. It is of interest to note that these perturbations constitute an invariant subspace for the evolution of $\tilde{u}(t)$. The following assumption and the subsequent theorem are fundamental to the later sections.

Assumption 1. The initial data $\tilde{u}_{0} \in \mathfrak{g}^{*}$ of the linearized equation (E) is kinematically accessible from the equilibrium state $u \in \mathfrak{g}^{*}$, i.e.,

$$
\exists \zeta_{0} \in \mathfrak{g} \text { such that } \tilde{u}_{0}=\mathcal{A} \zeta_{0} .
$$

Theorem 2. Under the assumption $(\mathrm{A})$, the solution $\tilde{u}(t)$ of $(\mathrm{E})$ remains kinematically accessible to $u \in \mathfrak{g}^{*} ; \tilde{u}(t)=\mathcal{A} \zeta(t)$ for all $t>0$ where $\zeta(t)$ is a solution of the adjoint problem

$$
\partial_{t} \zeta=\left(\mathcal{H} \mathcal{A}-\mathcal{B}^{*}\right) \zeta, \quad \zeta(0)=\zeta_{0}
$$

In addition, a symmetric quadratic form $\delta^{2} H: \mathfrak{g} \times \mathfrak{g} \rightarrow \mathbb{R}$ defined by

$$
\delta^{2} H:=-\langle(\mathcal{A H}+\mathcal{B}) \mathcal{A} \zeta, \zeta\rangle=\left\langle\tilde{u}, \partial_{t} \zeta\right\rangle
$$

is a constant of motion.

Proof. Using the Jacobi identity of the Lie bracket, $\mathcal{B} \mathcal{A}$ is proved to be symmetric $(\mathcal{B A})^{*}=$ $\mathcal{B A}$ as follows; for all $\zeta_{1}, \zeta_{2} \in \mathfrak{g}$,

$$
\begin{aligned}
\left\langle\mathcal{B} \mathcal{A} \zeta_{1}, \zeta_{2}\right\rangle & =-\left\langle u,\left[\left[\frac{\delta H}{\delta u}, \zeta_{2}\right], \zeta_{1}\right]\right\rangle, \\
& =\left\langle u,\left[\left[\zeta_{2}, \zeta_{1}\right], \frac{\delta H}{\delta u}\right]\right\rangle+\left\langle u,\left[\left[\zeta_{1}, \frac{\delta H}{\delta u}\right], \zeta_{2}\right]\right\rangle, \\
& =\left\langle\operatorname{ad}^{*}\left(\frac{\delta H}{\delta u}\right) u,\left[\zeta_{1}, \zeta_{2}\right]\right\rangle-\left\langle u,\left[\left[\frac{\delta H}{\delta u}, \zeta_{1}\right], \zeta_{2}\right]\right\rangle, \\
& =\left\langle\mathcal{B} \mathcal{A} \zeta_{2}, \zeta_{1}\right\rangle,
\end{aligned}
$$

where we used the equilibrium condition (11) in the last equality.

Let $\zeta(t)$ be a solution of $\left(\mathrm{E}^{*}\right)$. By operating $\mathcal{A}$ on both sides of $\left(\mathrm{E}^{*}\right)$, the same $\zeta(t)$ satisfies

$$
\partial_{t}(\mathcal{A} \zeta)=(\mathcal{A H}+\mathcal{B}) \mathcal{A} \zeta, \quad \mathcal{A} \zeta(0)=\mathcal{A} \zeta_{0},
$$

which implies that $\mathcal{A} \zeta(t)$ is the solution of $(\mathrm{E})$. Since $\mathcal{A H} \mathcal{A}+\mathcal{B} \mathcal{A}$ is symmetric, one can prove that

$$
\begin{aligned}
\partial_{t}\langle(\mathcal{A H} \mathcal{A}+\mathcal{B} \mathcal{A}) \zeta, \zeta\rangle & =\left\langle\partial_{t} \zeta,(\mathcal{A H} \mathcal{A}+\mathcal{B} \mathcal{A}) \zeta\right\rangle+\left\langle(\mathcal{A H} \mathcal{A}+\mathcal{B \mathcal { A }}) \zeta, \partial_{t} \zeta\right\rangle \\
& =\left\langle\partial_{t} \zeta, \mathcal{A} \partial_{t} \zeta\right\rangle+\left\langle\mathcal{A} \partial_{t} \zeta, \partial_{t} \zeta\right\rangle \\
& =0
\end{aligned}
$$


The proof of $(\mathcal{B A})^{*}=\mathcal{B A}$ and the subsequent claim, $\delta^{2} H=$ const., has already given in Ref. 2 with some different notations. It must be emphasized here that there is an explicit duality between the variables $\tilde{u}$ and $\zeta$. The solution of the linearized Lie-Poisson equation $(\mathrm{E})$ is virtually equivalent to that of the adjoint problem $\left(\mathrm{E}^{*}\right)$ via the mapping $\mathcal{A}$. This fact and the underlying assumption (A) are required for the second (kinematically accessible) variation $\delta^{2} H$ of $H$ to be invariant. We will refer to this $\delta^{2} H=\left\langle\tilde{u}, \partial_{t} \zeta\right\rangle$ as the energy of perturbation.

\section{WAVE ENERGY AND WAVE ACTION}

In the linear theory, it is useful to regard the perturbations $\tilde{u} \in \mathfrak{g}^{*}$ and $\zeta \in \mathfrak{g}$ as complex variables and invoke the Fourier-Laplace transform. In what follows, we will naturally identify $\mathfrak{g}^{*}$ as $\mathfrak{g}$ and extend them into a complex Hilbert space $L^{2}$. The inner product is then given by $\langle\overline{\tilde{u}}, \zeta\rangle$ for any $\tilde{u}, \zeta \in L^{2}$, where the bar $\left(^{-}\right)$denotes complex conjugate. By just multiplying the linearized systems by the imaginary unit $i$, we get Schrödinger-like equations,

$$
\begin{aligned}
i \partial_{t} \tilde{u} & =\mathcal{L} \tilde{u}, \quad \tilde{u}(0)=\tilde{u}_{0}, \\
i \partial_{t} \zeta=\mathcal{L}^{*} \zeta, & \zeta(0)=\zeta_{0} .
\end{aligned}
$$

where we defined two pure-imaginary operators, $\mathcal{L}:=i(\mathcal{A H}+\mathcal{B})$ and $\mathcal{L}^{*}:=i\left(\mathcal{H} \mathcal{A}-\mathcal{B}^{*}\right)$. Note that $\mathcal{L}^{*}$ is indeed the adjoint operator of $\mathcal{L}$ with respect to the $L^{2}$ inner product and the following important relation holds,

$$
\mathcal{L} \mathcal{A}=\mathcal{A} \mathcal{L}^{*} .
$$

Although the solutions of $\left(\mathrm{E}^{\prime}\right)$ and $\left(\mathrm{E}^{\prime *}\right)$ may look complex-valued in the Fourier-Laplace representation, remember that they must be always real as far as the initial data $\tilde{u}_{0}$ and $\zeta_{0}$ are real $\left(\overline{\tilde{u}_{0}}=\tilde{u}_{0}\right.$ and $\left.\overline{\zeta_{0}}=\zeta_{0}\right)$.

The linear waves and their frequencies respectively correspond to the eigenfunctions and the eigenvalues of the linear operator $\mathcal{L}$. Since fluids and plasmas have infinite degreeof-freedom, the spectrum of $\mathcal{L}$ generally includes the continuous spectrum as well as the point (or discrete) spectrum. We will discuss wave energy for both kinds of spectra in this section. However, it must be remarked that the spectral decomposition of the non-selfadjoint operator $\mathcal{L}$ in functional space is mathematically nontrivial. For example, the choice of the functional space as the domain of $\mathcal{L}$ depends on the smoothness of the equilibrium fields in a complicated manner. We leave this subtle problem for future works which would be more detailed and math-oriented. In this work, we shall utilize the Laplace transform in time and apply the hyperfunction theory [30], which can deal formally with the singularity of the resolvent operator, $(\Omega-\mathcal{L})^{-1}$, without consideration of the subtlety of the domain of $\mathcal{L}$.

Let $\mathrm{U}(\Omega)$ and $\mathrm{Z}(\Omega)(\Omega \in \mathbb{C})$ be the solutions of

$$
\begin{aligned}
(\Omega-\mathcal{L}) \mathrm{U}(\Omega) & =i \tilde{u}_{0}, \\
\left(\Omega-\mathcal{L}^{*}\right) \mathrm{Z}(\Omega) & =i \zeta_{0} .
\end{aligned}
$$

The spectrum $\operatorname{Sp}(\mathcal{L}) \subset \mathbb{C}$ of $\mathcal{L}$ is then characterized by the singularities of $\mathrm{U}(\Omega)$ on the complex $\Omega$-plane;

$$
\operatorname{Sp}(\mathcal{L})=\left\{\omega \in \mathbb{C}: U(\Omega)=i(\Omega-\mathcal{L})^{-1} \tilde{u}_{0} \text { is not regular at } \Omega=\omega\right\} .
$$


Similarly, the singularities of $Z(\Omega)$ correspond to the spectrum $\operatorname{Sp}\left(\mathcal{L}^{*}\right)$, which is generally known to be the complex conjugate of $\operatorname{Sp}(\mathcal{L}) ; \operatorname{Sp}(\mathcal{L})=\overline{\operatorname{Sp}\left(\mathcal{L}^{*}\right)}$. Note that $\mathrm{U}(\Omega)[$ or $\mathrm{Z}(\Omega)]$ is nothing but the double-sided Laplace transform of $\tilde{u}(t)$ [or $\zeta(t)]$, which is valid for all, positive and negative, times, namely,

$$
\begin{aligned}
& \mathrm{U}(\Omega)=\int_{0}^{\infty} \tilde{u}(t) e^{i \Omega t} d t \quad \text { for } \Im(\Omega)>\sup _{\omega \in \operatorname{Sp}(\mathcal{L})}[\Im(\omega)], \\
& \mathrm{U}(\Omega)=\int_{0}^{-\infty} \tilde{u}(t) e^{i \Omega t} d t \quad \text { for } \Im(\Omega)<\inf _{\omega \in \operatorname{Sp}(\mathcal{L})}[\Im(\omega)],
\end{aligned}
$$

where $\Im(\circ)$ denotes the imaginary part. The inverse of this transform is especially called the Dunford-Taylor integral (see Ref. 31).

When the initial data is restricted by the assumption (A), the resultant solution may not include all eigenmodes and, hence, its spectrum is possibly a subset of $\operatorname{Sp}(\mathcal{L})$, which will be denoted by $\sigma \subset \operatorname{Sp}(\mathcal{L})$.

Lemma 3. Let $\tilde{u}(t)$ be the solution of $(\mathrm{E})$ under the assumption $(\mathrm{A})$. Then, the spectrum $\sigma \subset \operatorname{Sp}(\mathcal{L})$ of $\tilde{u}(t)$, defined by

$$
\sigma=\left\{\omega \in \mathbb{C}: \mathcal{A Z}(\Omega)=i(\Omega-\mathcal{L})^{-1} \mathcal{A} \zeta_{0} \text { is not regular at } \Omega=\omega\right\},
$$

satisfies $\sigma \subset \operatorname{Sp}\left(\mathcal{L}^{*}\right)$ and $\sigma=\bar{\sigma}=-\bar{\sigma}=-\sigma$.

Proof. By operating $\mathcal{A}$ on the both sides of (19) and using the property $\mathcal{L} \mathcal{A}=\mathcal{A} \mathcal{L}^{*}$, we get

$$
(\Omega-\mathcal{L}) \mathcal{A Z}(\Omega)=i \mathcal{A} \zeta_{0}=i \tilde{u}_{0}
$$

and the Laplace transform of $\tilde{u}(t)$ turns out to be $\mathrm{U}(\Omega)=\mathcal{A Z}(\Omega)$. Now, $\sigma$ is identified as the set of $\Omega \in \mathbb{C}$ at which $\mathcal{A Z}(\Omega)$ is not regular for some $\zeta_{0}$. All singularities of $\mathcal{A Z}(\Omega)$ necessarily comes from that of $\mathrm{Z}(\Omega)$, which implies $\sigma \subset \operatorname{Sp}\left(\mathcal{L}^{*}\right)$.

(Proof of $\sigma=\bar{\sigma}$ ) For any $\omega \in \sigma$, there exist some $\zeta_{1}, \zeta_{2} \in L^{2}$ such that a function $f(\Omega):=$ $\left\langle\overline{\zeta_{1}},(\Omega-\mathcal{L})^{-1} i \mathcal{A} \zeta_{2}\right\rangle$ is not regular at $\Omega=\omega$. Using $\mathcal{L} \mathcal{A}=\mathcal{A} \mathcal{L}^{*}$ again, this function can be rewritten as

$$
f(\Omega)=\left\langle\overline{(\bar{\Omega}-\mathcal{L})^{-1} i \mathcal{A} \zeta_{1}}, \zeta_{2}\right\rangle
$$

Since this expression is not regular at $\Omega=\omega$ too, it proves that $\bar{\omega} \in \sigma$ and, hence, $\sigma=\bar{\sigma}$. (Proof of $\sigma=-\bar{\sigma})$ By taking the complex conjugate of $\left(\Omega-\mathcal{L}^{*}\right) \mathrm{Z}(\Omega)=i \zeta_{0}$ and then replacing $\Omega$ by $-\bar{\Omega}$, we get

$$
\left(\Omega-\mathcal{L}^{*}\right) \overline{\mathrm{Z}(-\bar{\Omega})}=i \zeta_{0}
$$

where we used $\overline{\mathcal{L}^{*}}=-\mathcal{L}^{*}$ and $\overline{\zeta_{0}}=\zeta_{0}$. It follows from (19) that $\mathrm{Z}(\Omega)$ satisfies $\mathrm{Z}(\Omega)=\overline{\mathrm{Z}(-\bar{\Omega})}$ and, hence, $\sigma=-\bar{\sigma}$.

The spectrum of HD and MHD waves is composed of the point spectrum $\sigma_{p}$ and the continuous spectrum $\sigma_{c} ; \sigma=\sigma_{p} \cup \sigma_{c}$. In this paper, we assume that the continuous spectrum lies on the real axis, $\sigma_{c} \subset \mathbb{R}$, as is common with ideal fluids and plasmas. In addition, all point spectra are supposed to be semi-simple for simplicity. 
The solution $\tilde{u}(t)$ is expressed by the Dunford-Taylor integral (or the double-sided inverse Laplace transform),

$$
\tilde{u}(t)=-\frac{1}{2 \pi} \oint_{\Gamma(\sigma)} \mathrm{U}(\Omega) e^{-i \Omega t} \mathrm{~d} \Omega
$$

where the path of integration $\Gamma(\sigma)$ encircles the all spectrum $\sigma$ in the counterclockwise direction as illustrated in Figure 1(a). This $\Gamma(\sigma)$ can be analytically deformed into the neighborhood of each point and continuous spectra as in Figure 1(b). Since the semi-simple point spectra $\omega_{j} \in \sigma_{p}, j=1,2, \ldots$, appear as the poles of the first order in the $\Omega$-plane, the residue theorem yields the corresponding eigenfunctions of $\mathcal{L}$ and $\mathcal{L}^{*}$, denoted respectively by

$$
\begin{aligned}
\hat{\tilde{u}}\left(\omega_{j}\right) & :=-\frac{1}{2 \pi} \oint_{\Gamma\left(\omega_{j}\right)} \mathrm{U}(\Omega) \mathrm{d} \Omega, \\
\hat{\zeta}\left(\omega_{j}\right) & :=-\frac{1}{2 \pi} \oint_{\Gamma\left(\omega_{j}\right)} \mathrm{Z}(\Omega) \mathrm{d} \Omega .
\end{aligned}
$$

As for the continuous spectrum $\sigma_{c} \subset \mathbb{R}$, the path of integration is deformed into the two paths that run parallel to $\sigma_{c}$ at the slightly upper and lower sides;

$$
-\frac{1}{2 \pi} \oint_{\Gamma\left(\sigma_{c}\right)} \mathrm{U}(\Omega) \mathrm{d} \Omega=\lim _{\varepsilon \rightarrow 0} \frac{1}{2 \pi} \int_{\sigma_{c}}[\mathrm{U}(\omega+i \varepsilon)-\mathrm{U}(\omega-i \varepsilon)] \mathrm{d} \omega .
$$

Hence, it is reasonable to define the generalized eigenfunction for $\omega \in \sigma_{c}$ by

$$
\begin{aligned}
\hat{\tilde{u}}(\omega) & :=\frac{1}{2 \pi}[\mathrm{U}(\omega+i 0)-\mathrm{U}(\omega-i 0)], \\
\hat{\zeta}(\omega) & :=\frac{1}{2 \pi}[\mathrm{Z}(\omega+i 0)-\mathrm{Z}(\omega-i 0)] .
\end{aligned}
$$

This definition of $\hat{\tilde{u}}(\omega)$ is consistent with the Fourier transform of $\tilde{u}(t)$ according to Sato's hyperfunction theory [30] (see also the Appendix of Ref. 32). The eigenfunction $\hat{\tilde{u}}(\omega)$ for the continuous spectrum $\omega \in \sigma_{c}$ is therefore a generalized (or singular) function. This fact has been pointed out in many literatures; for example, see Case [33, 34], Sedláček [35] and Tataronis [36].

Since we have $\mathrm{U}(\Omega)=\mathcal{A Z}(\Omega)$ under the assumption $(\mathrm{A})$, the following relation is satisfied;

$$
\hat{\tilde{u}}(\omega)=\mathcal{A} \hat{\zeta}(\omega) \text { for } \omega \in \sigma_{p} \cup \sigma_{c} .
$$

While $\mathcal{A}$ is usually a differential operator, this relation holds even for $\omega \in \sigma_{c}$ in the sense of hyperfunction (note that hyperfunctions are infinitely differentiable).

We must remark that the eigenfunctions we defined above are not normalized, that is, the amplitudes of the eigenmodes are included in the definition of $\{\hat{\tilde{u}}(\omega): \omega \in \sigma\}$. Correctly speaking, they must be called the projections of $\tilde{u}_{0}$ into the eigenspaces. Nevertheless, the normalization does not seem to be essential in the present case because the eigenfunctions are non-orthogonal to each other due to the non-selfadjointness of $\mathcal{L}$ and, furthermore, they may not be square integrable as for the continuous spectrum.

The wave action defined below, without normalizing the amplitude, is found to be more insightful. Although the eigenfunctions $\left\{\hat{\tilde{u}}\left(\omega_{j}\right)\right\}$ for point spectra $\omega_{j} \in \sigma_{p}$ are non-orthogonal, 

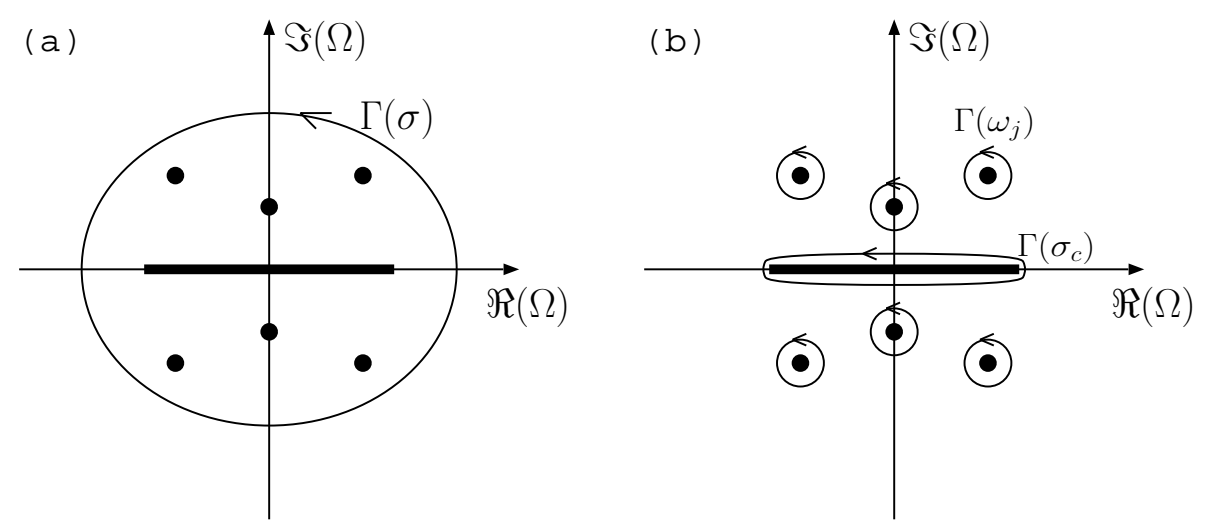

FIG. 1: Path of the Dunford integration

the dual basis is provided by the eigenfunctions $\left\{\hat{\zeta}\left(\omega_{j}\right)\right\}$ of the adjoint operator $\mathcal{L}^{*}$. As is well known from linear algebra, we have the following orthogonality,

$$
\left\langle\overline{\hat{\zeta}\left(\overline{\omega_{j}}\right)}, i \mathcal{A} \hat{\zeta}\left(\omega_{l}\right)\right\rangle=\left\langle\overline{\zeta_{0}}, i \mathcal{A} \hat{\zeta}\left(\omega_{j}\right)\right\rangle \delta_{j l}=: \mu_{p}\left(\omega_{j}\right) \delta_{j l} \quad \text { for } \omega_{j}, \omega_{l} \in \sigma_{p}
$$

where $\delta_{j l}$ denotes the Kronecker delta. We call the quantities $\mu_{p}\left(\omega_{j}\right), j=1,2, \ldots$, wave actions since they will prove to be the action variables of the eigenmodes. The magnitude of $\mu_{p}\left(\omega_{j}\right)$ depends on the square of the corresponding modal amplitude and measures the activity of the wave. The sign of $\mu_{p}\left(\omega_{j}\right)$ can be either positive or negative (if the symmetric operator, $i \mathcal{A}$, is indefinite), which is of particular interest in the bifurcation theory of Hamiltonian systems $[5,6]$. In this work, we also define the action $\mu_{c}(\omega)$ for the continuous spectrum similarly as follows.

$$
\left\langle\overline{\hat{\zeta}(\omega)}, i \mathcal{A} \hat{\zeta}\left(\omega^{\prime}\right)\right\rangle=\left\langle\overline{\zeta_{0}}, i \mathcal{A} \hat{\zeta}(\omega)\right\rangle \delta\left(\omega-\omega^{\prime}\right)=: \mu_{c}(\omega) \delta\left(\omega-\omega^{\prime}\right) \text { for } \omega, \omega^{\prime} \in \sigma_{c}
$$

where $\delta\left(\omega-\omega^{\prime}\right)$ denotes the Dirac delta function. While this definition looks straightforward, the treatment of the hyperfunctions such as $\hat{\zeta}(\omega)$ and $\delta\left(\omega-\omega^{\prime}\right)$ requires some mathematical justification. The orthogonality between singular eigenfunctions has been claimed in several works $[15,24,33]$, while the proof requires laborious calculations depending on the problems. Here, we present a lemma that generally ensures this orthogonality as follows.

Lemma 4. Suppose that the operator $\mathcal{L}$ has a real spectrum $\sigma \subset \mathbb{R}$. Let $\tilde{u}(t)$ and $\zeta(t)$ be, respectively, the solutions of $\left(\mathrm{E}^{\prime}\right)$ and $\left(\mathrm{E}^{\prime *}\right)$. Then, the generalized eigenfunctions, $\hat{\tilde{u}}(\omega)$ and $\hat{\zeta}(\omega)$, defined in (30) and (31) satisfy

$$
\left\langle\overline{\hat{\tilde{u}}(\omega)}, \hat{\zeta}\left(\omega^{\prime}\right)\right\rangle=\delta\left(\omega-\omega^{\prime}\right)\left\langle\overline{\tilde{u}_{0}}, \hat{\zeta}(\omega)\right\rangle=\delta\left(\omega-\omega^{\prime}\right)\left\langle\overline{\hat{\tilde{u}}(\omega)}, \zeta_{0}\right\rangle
$$

for $\omega, \omega^{\prime} \in \sigma$.

The proof of this lemma according to the hyperfunction theory is given in the appendix B.

The above spectral decomposition and the orthogonality theorem are applied to the theorem 2, which enables us to define wave energy as follows. 
Theorem 5. Let $\tilde{u}(t)$ be a solution of $(\mathrm{E})$ under the assumption (A). If the spectrum $\sigma$ is composed of semi-simple point spectra $\sigma_{p}=\left\{\omega_{j} \in \mathbb{C}: j=1,2, \ldots\right\}$ and real continuous spectrum $\sigma_{c} \subset \mathbb{R}$, the solution is represented by

$$
\tilde{u}(t)=\sum_{\omega_{j} \in \sigma_{p}} \hat{\tilde{u}}\left(\omega_{j}\right) e^{-i \omega_{j} t}+\int_{\sigma_{c}} \hat{\tilde{u}}(\omega) e^{-i \omega t} \mathrm{~d} \omega,
$$

and the energy of perturbation $\delta^{2} H=\left\langle\tilde{u}(t), \partial_{t} \zeta(t)\right\rangle=$ const. is decomposed into the energy of waves,

$$
\delta^{2} H=\sum_{\omega_{j} \in \sigma_{p}} \Re\left[\omega_{j} \mu_{p}\left(\omega_{j}\right)\right]+\int_{\sigma_{c}} \omega \mu_{c}(\omega) \mathrm{d} \omega,
$$

where the real part $(\Re)$ needs to be taken when $\omega_{j}$ is complex.

Thus, we saw that the wave energy is generally expressed by the multiplication of the wave frequency and the wave action, regardless of whether the wave frequency (namely, the spectrum) is discrete or continuous. This fact is originally stemming from the assumption (A), under which the linear perturbations behave like canonical variables.

In general, the point spectra $\left\{\omega_{j}\right\}$ may not be semi-simple if some of them are degenerated. Since the generator $\mathcal{L}$ of $\left(\mathrm{E}^{\prime}\right)$ is non-selfadjoint, such multiple eigenvalues may constitute a nilpotent in the Jordan canonical form of $\mathcal{L}$ (see, for example, Ref. 31), where $\mathrm{U}(\Omega)$ includes a multiple pole $\left(\Omega-\omega_{j}\right)^{-n}, n \geq 2$, and the solution exhibits secular growth $t^{n-1} e^{-i \omega_{j} t}$. As was discussed by MacKay [6] in finite-dimensional Hamiltonian systems, this situation can be associated with marginal state of the Krein collision. However, Jordan's theory is not established for a continuous spectrum of non-selfadjoint operator. The solution stemming from the hydrodynamic continuous spectrum is still nontrivial when some degeneracy of spectra occurs on it $[32,37]$. In Theorem 5 , this difficulty remains in the singularity of $\tilde{\tilde{u}}(\omega)$ for $\omega \in \sigma_{c}$ which is highly complicated in practice, and the counterpart of Jordan's theory is yet to be explored for infinite-dimensional systems.

Before ending this section, we introduce another expression for the wave action which manifests a link with the dispersion relation. Let us define a linear operator $\mathcal{E}(\Omega): L^{2} \rightarrow L^{2}$ by

$$
\mathcal{E}(\Omega)=i(\Omega-\mathcal{L}) \mathcal{A}
$$

with $\Omega \in \mathbb{C}$ being a complex parameter. A symmetric property $\mathcal{E}^{*}(\Omega)=\mathcal{E}(\bar{\Omega})$ follows from $\mathcal{A}=-\mathcal{A}^{*}$ and $\mathcal{L} \mathcal{A}=\mathcal{A} \mathcal{L}^{*}$. In this work, we will refer to the equation (24) or

$$
\mathcal{E}(\Omega) Z(\Omega)=-\tilde{u}_{0},
$$

as symmetric response equation, for the solution $\mathrm{Z}(\Omega)$ represents the frequency response to some (kinematically accessible) initial data $\tilde{u}_{0}$. If our problem was a system of finite degrees of freedom, the space $L^{2}$ would be a finite-dimensional vector space and $\mathcal{E}(\Omega)$ be a matrix of numbers. Then, the dispersion relation would be given by the determinant, $D(\Omega)=\operatorname{det}|\mathcal{E}(\Omega)|=0$. However, since we are considering the system of infinite degrees of freedom, $\mathcal{E}(\Omega)$ is generally a differential operator and the continuous spectrum shows up. It is no longer possible to obtain the dispersion relation algebraically. We, therefore, introduce generalized dispersion relation as follows. 
Proposition 6. Define the generalized dispersion relation $D: \mathbb{C} \times L^{2} \times L^{2} \rightarrow \mathbb{C}$ as

$$
D\left(\Omega, \zeta_{1}, \zeta_{2}\right):=\left\langle\overline{\zeta_{1}}, \mathcal{E}(\Omega) \zeta_{2}\right\rangle
$$

Then, the wave actions for a semi-simple point spectrum $\omega_{j} \in \sigma_{p}$ and real continuous spectrum $\omega \in \sigma_{c}$ are, respectively, given by

$$
\begin{aligned}
\mu_{p}\left(\omega_{j}\right) & =\frac{1}{2 \pi i} \oint_{\Gamma\left(\omega_{j}\right)} D(\Omega, \mathbf{Z}(\bar{\Omega}), \mathrm{Z}(\Omega)) \mathrm{d} \Omega \\
\mu_{c}(\omega) & =\frac{i}{2 \pi}\left[\lim _{\Omega \rightarrow \omega+i 0} D(\Omega, \mathrm{Z}(\bar{\Omega}), \mathrm{Z}(\Omega))-\lim _{\Omega \rightarrow \omega-i 0} D(\Omega, \mathrm{Z}(\bar{\Omega}), \mathrm{Z}(\Omega))\right] .
\end{aligned}
$$

While this proposition is straightforward from the definitions of $\mu_{p}$ and $\mu_{c}$, the above expressions will play an important role in the subsequent sections. Using a trivial relation $\partial \mathcal{E} / \partial \Omega=i \mathcal{A}$, one may write

$$
\mu_{p}\left(\omega_{j}\right)=\left\langle\overline{\hat{\zeta}\left(\overline{\omega_{j}}\right)}, \frac{\partial \mathcal{E}}{\partial \Omega} \hat{\zeta}\left(\omega_{j}\right)\right\rangle=\frac{\partial D}{\partial \Omega}\left(\omega_{j}, \hat{\zeta}\left(\overline{\omega_{j}}\right), \hat{\zeta}\left(\omega_{j}\right)\right),
$$

which is similar to the conventional formula (2). This relationship between the wave action and the dispersion relation will turn out to be universal for a point spectrum. On the contrary, another rule (42) must be applied to the case of a continuous spectrum. Indeed, the result of Balmforth and Morrison [15] will be recovered by using (42) in Sec. V.

\section{MAGNETOHYDRODYNAMIC WAVES}

In this section, we apply the general theorems discussed so far to the linearized MHD equation. The theorem 2 is worthwhile not only for formulating the energy of perturbation but also for reducing the equation to a simpler one. Note that, under the assumption (A), the linearized equation (E) for the MHD case can be reduced to the equation derived by Frieman and Rotenberg [19], which is easier to be solved especially when the mean flow is absent (or uniform). Let $u=(\boldsymbol{M}, \boldsymbol{B}, \rho, s)^{T}$ denote the mean fields and $\boldsymbol{M}=\rho \boldsymbol{v}$. Since the theorem 2 assures $\tilde{u}(t)=\mathcal{A} \zeta(t)$ for all $t>0$, the corresponding relations, similar to (7)-(10), holds between $\tilde{u}=(\tilde{\boldsymbol{M}}, \tilde{\boldsymbol{B}}, \tilde{\rho}, \tilde{s})^{T}$ and $\zeta=(\boldsymbol{\xi}, \boldsymbol{\eta}, \alpha, \beta)^{T}$. It is remarkable that the $\boldsymbol{\xi}$-component of $\left(\mathrm{E}^{*}\right)$ can be written as

$$
\partial_{t} \boldsymbol{\xi}+(\boldsymbol{v} \cdot \nabla) \boldsymbol{\xi}-(\boldsymbol{\xi} \cdot \nabla) \boldsymbol{v}=\mathcal{V}(\zeta)
$$

where we defined an operator $\mathcal{V}: \mathfrak{g} \rightarrow \mathfrak{X}$, for later use, as

$$
\mathcal{V}(\zeta):=\boldsymbol{\xi} \times(\nabla \times \boldsymbol{v})-\frac{1}{\rho} \boldsymbol{B} \times(\nabla \times \boldsymbol{\eta})-\nabla(\boldsymbol{\xi} \cdot \boldsymbol{v}+\alpha)+\frac{\beta}{\rho} \nabla s
$$

By noting that $\tilde{\boldsymbol{v}}(t)=\mathcal{V}(\zeta(t))$ holds according to $\tilde{u}(t)=\mathcal{A} \zeta(t)$ [see $(7)]$, the variable $\boldsymbol{\xi}(t) \in \mathfrak{X}$ automatically agrees with the conventional definition of the Lagrangian displacement field, which is the displacement vector field of the fluid particle orbits [19]. Therefore, the relations $\tilde{\boldsymbol{B}}=\nabla \times(\boldsymbol{\xi} \times \boldsymbol{B}), \tilde{\rho}=-\nabla \cdot(\rho \boldsymbol{\xi})$ and $\tilde{s}=-\boldsymbol{\xi} \cdot \nabla s$ implies that magnetic field, mass and entropy are frozen in the fluid particle motion, and the topological structure of the 
mean fields is preserved for this perturbation [38]. Substituting these relations into the $\tilde{M}$ component of (E), we can eliminate $\boldsymbol{\eta}, \alpha$ and $\beta$ from the evolution equation and reproduce the Frieman-Rotenberg equation for $\boldsymbol{\xi} \in \mathfrak{X}$ with restricted initial data;

$$
\left\{\begin{aligned}
& \rho \partial_{t}^{2} \boldsymbol{\xi}+2 \rho(\boldsymbol{v} \cdot \nabla) \partial_{t} \boldsymbol{\xi}=\mathcal{F} \boldsymbol{\xi}, \\
&\left(\partial_{t} \boldsymbol{\xi}\right)(0)=-2(\boldsymbol{v} \cdot \nabla) \boldsymbol{\xi}_{0}-\boldsymbol{v} \times\left(\nabla \times \boldsymbol{\xi}_{0}\right) \\
&-\rho^{-1} \boldsymbol{B} \times\left(\nabla \times \boldsymbol{\eta}_{0}\right)-\nabla \alpha_{0}+\rho^{-1} \beta_{0} \nabla s \\
& \boldsymbol{\xi}(0)= \boldsymbol{\xi}_{0} .
\end{aligned}\right.
$$

The so-called Force operator, $\mathcal{F}: \mathfrak{X} \rightarrow \mathfrak{X}$, is a selfadjoint operator derived in Ref. 19 as

$$
\begin{aligned}
\mathcal{F} \boldsymbol{\xi}:=\nabla\left(\rho c_{s}^{2} \nabla\right. & \cdot \boldsymbol{\xi}+\boldsymbol{\xi} \cdot \nabla p-\boldsymbol{B} \cdot \boldsymbol{Q})+(\boldsymbol{B} \cdot \nabla) \boldsymbol{Q}+(\boldsymbol{Q} \cdot \nabla) \boldsymbol{B} \\
& +[\nabla \cdot(\rho \boldsymbol{\xi})](\boldsymbol{v} \cdot \nabla) \boldsymbol{v}-\rho(\boldsymbol{v} \cdot \nabla) \boldsymbol{R}-\rho(\boldsymbol{R} \cdot \nabla) \boldsymbol{v},
\end{aligned}
$$

where $\boldsymbol{Q}=\nabla \times(\boldsymbol{\xi} \times \boldsymbol{B}), \boldsymbol{R}=(\boldsymbol{v} \cdot \nabla) \boldsymbol{\xi}-(\boldsymbol{\xi} \cdot \nabla) \boldsymbol{v}$ and $c_{s}=\sqrt{\partial p / \partial \rho}$. Our assumption (A) based on the Hamiltonian structure indicates that the initial data for $\partial_{t} \boldsymbol{\xi}$ should be somewhat restricted as above such that the perturbation remains kinematically accessible. If $\left(\partial_{t} \boldsymbol{\xi}\right)(0)$ were arbitrary, no restriction would be imposed on the initial velocity perturbation $\tilde{\boldsymbol{v}}_{0}$, which would violate the conservation laws related to the circulation theorem [20]. Evidently, the cross helicity $\int \boldsymbol{v} \cdot \boldsymbol{B} \mathrm{d}^{3} x$ [or the helicity $\int \boldsymbol{v} \cdot(\nabla \times \boldsymbol{v}) \mathrm{d}^{3} x$ in the HD case] would be perturbed by it.

The energy of kinematically accessible perturbation $\delta^{2} H$, defined by Theorem 2, also agrees with the expression of energy derived in Ref. 19 (see Ref. 20 for detailed comparison). The positive definiteness of the potential energy, $U(\boldsymbol{\xi})=-\int \boldsymbol{\xi} \cdot \mathcal{F} \boldsymbol{\xi} \mathrm{d}^{3} x \geq 0$, gives a sufficient stability condition. This criterion works very well for static equilibria $(\boldsymbol{v}=0)$, for which (46) is analogous to Newton's second law. The energy principle [39] claims that a static equilibrium is stable if and only if the potential energy is positive definite. Linear instabilities always emerge from the zero eigenvalue of the selfadjoint operator $\mathcal{F}$, whose spectral decomposition can be discussed by the well-established methods (like the Von Neumann theorem) in the quantum mechanics.

However, Hameiri [20, 40] showed that the potential energy (and also the total energy $\left.\delta^{2} H\right)$ of perturbation often turns out to be indefinite in the presence of the basic flow $\boldsymbol{v}$. The basic flow $\boldsymbol{v}$, moreover, plays the role of the gyroscopic term [27] $2 \rho(\boldsymbol{v} \cdot \nabla) \partial_{t} \boldsymbol{\xi}$ in (46), which allows the existence of neutrally stable modes with negative energy. These facts imply that the energy stability criterion is rather difficult to be met for flowing plasmas. It then seems to be important to evaluate the wave energy (or action) for the purpose of predicting and understanding various instabilities.

Now, let us consider the wave energy for the MHD case. The reduction to the FriemanRotenberg equation (46) is similarly applicable to the symmetric response equation (39). By denoting the Laplace transform of $\boldsymbol{\xi}(t)$ by $\boldsymbol{\Xi}(\Omega)$, the first row of (19) reads

$$
\Omega \boldsymbol{\Xi}(\Omega)+i(\boldsymbol{v} \cdot \nabla) \boldsymbol{\Xi}(\Omega)-i(\boldsymbol{\Xi}(\Omega) \cdot \nabla) \boldsymbol{v}-i \mathcal{V}(\mathbf{Z}(\Omega))=i \boldsymbol{\xi}_{0}
$$

Using this expression, one can eliminate $\mathcal{V}(Z(\Omega))$ from the first row of (39). The resultant equation of $\boldsymbol{\Xi}(\Omega)$ is nothing but the Laplace-transformed Frieman-Rotenberg equation,

$$
\mathcal{E}_{\mathrm{FR}}(\Omega) \Xi(\Omega)=-\mathbf{m}_{0}(\Omega),
$$


where the operator $\mathcal{E}_{\mathrm{FR}}(\Omega)$ is defined by

$$
\mathcal{E}_{\mathrm{FR}}(\Omega) \boldsymbol{\Xi}(\Omega):=\Omega^{2} \rho \boldsymbol{\Xi}(\Omega)+2 i \Omega \rho(\boldsymbol{v} \cdot \nabla) \boldsymbol{\Xi}(\Omega)+\mathcal{F} \boldsymbol{\Xi}(\Omega) .
$$

The right hand side of (49) has a nontrivial expression,

$$
\begin{aligned}
\mathbf{m}_{0}(\Omega) & :=\rho\left[\mathcal{V}\left(\zeta_{0}\right)-i \Omega \boldsymbol{\xi}_{0}+(\boldsymbol{v} \cdot \nabla) \boldsymbol{\xi}_{0}+\left(\boldsymbol{\xi}_{0} \cdot \nabla\right) \boldsymbol{v}\right] \\
& =-i \Omega \rho \boldsymbol{\xi}_{0}-\rho \boldsymbol{v} \times\left(\nabla \times \boldsymbol{\xi}_{0}\right)-\boldsymbol{B} \times\left(\nabla \times \boldsymbol{\eta}_{0}\right)-\rho \nabla \alpha_{0}+\beta_{0} \nabla s
\end{aligned}
$$

reflecting that the initial data was restricted by the assumption (A).

Since the operator $\mathcal{E}_{\mathrm{FR}}(\Omega)$ again satisfies $\mathcal{E}_{\mathrm{FR}}^{*}(\Omega)=\mathcal{E}_{\mathrm{FR}}(\bar{\Omega})$ in terms of the inner bracket $\int \bar{o} \cdot \circ d^{3} x$ of the reduced functional space, one can regard (49) as a new symmetric response equation and define

$$
D_{\mathrm{FR}}\left(\Omega, \boldsymbol{\xi}_{1}, \boldsymbol{\xi}_{2}\right)=\int \overline{\boldsymbol{\xi}}_{1} \cdot \mathcal{E}_{\mathrm{FR}}(\Omega) \boldsymbol{\xi}_{2} \mathrm{~d}^{3} x
$$

Theorem 7. In the MHD case, the wave action is represented by

$$
\begin{aligned}
\mu_{p}\left(\omega_{j}\right) & =i \int \overline{\hat{\boldsymbol{\xi}}\left(\overline{\omega_{j}}\right)} \cdot \mathbf{m}_{0}\left(\omega_{j}\right) \mathrm{d}^{3} x \\
& =\frac{\partial D_{\mathrm{FR}}}{\partial \Omega}\left(\omega_{j}, \hat{\boldsymbol{\xi}}\left(\overline{\omega_{j}}\right), \hat{\boldsymbol{\xi}}\left(\omega_{j}\right)\right) \\
& =\int \overline{\hat{\boldsymbol{\xi}}\left(\overline{\omega_{j}}\right)} \cdot 2 \rho\left[\omega_{j} \hat{\boldsymbol{\xi}}\left(\omega_{j}\right)+i(\boldsymbol{v} \cdot \nabla) \hat{\boldsymbol{\xi}}\left(\omega_{j}\right)\right] \mathrm{d}^{3} x .
\end{aligned}
$$

for a semi-simple point spectrum $\omega_{j} \in \sigma_{p}$ and

$$
\mu_{c}(\omega)=i \int \overline{\hat{\boldsymbol{\xi}}(\omega)} \cdot \mathbf{m}_{0}(\omega) \mathrm{d}^{3} x
$$

for a real continuous spectrum $\omega \in \sigma_{c} \subset \mathbb{R}$, where the eigenfunction $\hat{\boldsymbol{\xi}}\left(\omega_{j}\right)$ and the generalized eigenfunction $\hat{\boldsymbol{\xi}}(\omega)$ of the system (46) are generated by $\boldsymbol{\Xi}(\Omega)$ in the same manner as, respectively, (28) and (30).

Proof. It is essential to notice the following relation,

$$
\begin{aligned}
& \int \overline{\boldsymbol{\Xi}(\bar{\Omega})} \cdot \mathbf{m}_{0}(\Omega) d^{3} x=\int \overline{\boldsymbol{\Xi}(\bar{\Omega})} \cdot \rho\left[\mathcal{V}\left(\zeta_{0}\right)-i \Omega \boldsymbol{\xi}_{0}+(\boldsymbol{v} \cdot \nabla) \boldsymbol{\xi}_{0}+\left(\boldsymbol{\xi}_{0} \cdot \nabla\right) \boldsymbol{v}\right] \mathrm{d}^{3} x, \\
& =\int \overline{\boldsymbol{\Xi}(\bar{\Omega})} \cdot \rho\left[\mathcal{V}\left(\zeta_{0}\right)-\boldsymbol{\xi}_{0} \times(\nabla \times \boldsymbol{v})\right] \mathrm{d}^{3} x \\
& +\int \overline{[i \bar{\Omega} \boldsymbol{\Xi}(\bar{\Omega})-(\boldsymbol{v} \cdot \nabla) \boldsymbol{\Xi}(\bar{\Omega})+(\boldsymbol{\Xi}(\bar{\Omega}) \cdot \nabla) \boldsymbol{v}]} \cdot \rho \boldsymbol{\xi}_{0} \mathrm{~d}^{3} x \\
& \text { (integration by parts) } \\
& =\left\langle\overline{\mathrm{Z}(\bar{\Omega})}, \mathcal{A} \zeta_{0}\right\rangle-\int \rho\left|\boldsymbol{\xi}_{0}\right|^{2} \mathrm{~d}^{3} x, \quad \text { (use of (48) and integration by parts) }
\end{aligned}
$$

or, equivalently,

$$
D_{\mathrm{FR}}(\Omega, \boldsymbol{\Xi}(\bar{\Omega}), \boldsymbol{\Xi}(\Omega))=D(\Omega, \mathrm{Z}(\bar{\Omega}), \mathrm{Z}(\Omega))+\int \rho\left|\boldsymbol{\xi}_{0}\right|^{2} \mathrm{~d}^{3} x
$$


One may, therefore, replace $D(\Omega, \mathrm{Z}(\bar{\Omega}), \mathrm{Z}(\Omega))$ by $D_{\mathrm{FR}}(\Omega, \boldsymbol{\Xi}(\bar{\Omega}), \boldsymbol{\Xi}(\Omega))$ in the formulae (41) and (42), because the term $\int \rho\left|\boldsymbol{\xi}_{0}\right|^{2} d^{3} x$ independent of $\Omega$ does not affect the results. Since $\mathbf{m}_{0}(\Omega)$ depends on $\Omega$ analytically, these formulae yield (54) and (56) respectively.

To derive another expression (55), we invoke the fact that $\boldsymbol{\Xi}(\Omega)$ has semi-simple poles at $\Omega=\omega_{j}$ and $\Omega=\bar{\omega}_{j}$ (from Lemma 3 ). The Laurent expansion at these points must be

$$
\boldsymbol{\Xi}(\Omega)=\frac{i \hat{\boldsymbol{\xi}}\left(\omega_{j}\right)}{\Omega-\omega_{j}}+\ldots \quad \text { and } \quad \boldsymbol{\Xi}(\Omega)=\frac{i \hat{\boldsymbol{\xi}}\left(\overline{\omega_{j}}\right)}{\Omega-\bar{\omega}_{j}}+\ldots,
$$

where the $\operatorname{dots}(\ldots)$ represent the analytic parts of expansions. By expanding $\mathcal{E}_{\mathrm{FR}}(\Omega)$ also at $\Omega=\omega_{j}$, we get

$$
\begin{aligned}
& D_{\mathrm{FR}}(\Omega, \boldsymbol{\Xi}(\bar{\Omega}), \boldsymbol{\Xi}(\Omega))=\left\langle\overline{\frac{\hat{\boldsymbol{\xi}}\left(\overline{\omega_{j}}\right)}{\bar{\Omega}-\overline{\omega_{j}}}},\left[\mathcal{E}_{\mathrm{FR}}\left(\omega_{j}\right)+\left(\Omega-\omega_{j}\right) \frac{\partial \mathcal{E}_{\mathrm{FR}}}{\partial \Omega}\left(\omega_{j}\right)\right] \frac{\hat{\boldsymbol{\xi}}\left(\omega_{j}\right)}{\Omega-\omega_{j}}\right\rangle+\ldots, \\
& =\left\langle\overline{\hat{\boldsymbol{\xi}}\left(\overline{\omega_{j}}\right)}, \frac{\partial \mathcal{E}_{\mathrm{FR}}}{\partial \Omega}\left(\omega_{j}\right) \hat{\boldsymbol{\xi}}\left(\omega_{j}\right)\right\rangle \frac{1}{\Omega-\omega_{j}}+\ldots,
\end{aligned}
$$

where we used the fact that $\hat{\boldsymbol{\xi}}\left(\omega_{j}\right)$ is an eigenfunction, $\mathcal{E}_{\mathrm{FR}}\left(\omega_{j}\right) \hat{\boldsymbol{\xi}}\left(\omega_{j}\right)=0$. By putting this form into (41), the residue theorem leads to the required result (55).

Since both $\mathcal{E}_{\mathrm{FR}}(\Omega)$ and $\mathbf{m}_{0}(\Omega)$ are regular with respect to $\Omega$, the equation (49) and the related dispersion relation $D_{\mathrm{FR}}$ can be used anytime in place of (39). The wave energy for a point spectrum $\omega_{j} \in \sigma_{p}$ is now written by

$$
\omega_{j} \frac{\partial D_{\mathrm{FR}}}{\partial \Omega}\left(\omega_{j}, \hat{\boldsymbol{\xi}}\left(\bar{\omega}_{j}\right), \hat{\boldsymbol{\xi}}\left(\omega_{j}\right)\right)
$$

which is similar to (2). Note that the differentiation of $D_{\mathrm{FR}}$ with respect to $\Omega$ must be done before substituting the eigenvalue and the eigenfunctions. The formula (55) is more useful than (54) because it is symmetric and independent of initial data. For the continuous spectrum, however, the same formula does not seem to be applicable. The singularity of $\Xi(\Omega)$ at $\Omega=\omega \in \sigma_{c}$ is far from a simple pole and varies depending on the profiles of mean fields. We will seek the counterpart of the formula (55) for the continuous spectrum by assuming a simple geometry later.

It is obvious from (55) that, in the absence of basic flow $(\boldsymbol{v}=0)$, the wave action of any neutrally stable wave $\omega_{j} \in \mathbb{R}$ is simply $\mu_{p}\left(\omega_{j}\right)=2 \omega_{j} \int \rho\left|\hat{\boldsymbol{\xi}}\left(\omega_{j}\right)\right|^{2} \mathrm{~d}^{3} x$. Since the sign of the wave action corresponds to that of $\omega_{j}$, any linear instability occurs through the zero eigenvalue $\omega_{j}=0$ (static bifurcation). In other words, the Hopf bifurcation in fluids and plasmas is necessarily attributed to the presence of basic flow.

\section{A. Slab equilibria}

Further reduction of variables can be performed if the equilibrium state has a specific symmetry. As the simplest (but fundamental) example, we restrict our analysis to the slab geometry, i.e. the equilibrium is inhomogeneous only in the $x$ direction as follows,

$$
\boldsymbol{v}=\left(0, v_{y}(x), v_{z}(x)\right), \quad \boldsymbol{B}=\left(0, B_{y}(x), B_{z}(x)\right), \quad \rho(x) \quad \text { and } \quad s(x)
$$


on a bounded domain $x \in\left[x_{1}, x_{2}\right]$, where the boundary walls are located at $x=x_{1}, x_{2}$ and both flow and magnetic field are always tangential to them. Without external force, this is indeed an equilibrium state if the total pressure $p_{\text {total }}:=p(\rho, s)+B^{2} / 2$ satisfies

$$
p_{\text {total }}^{\prime}(x)=0
$$

where the prime $\left({ }^{\prime}\right)$ denotes the $x$-derivative of the equilibrium fields.

For fully three-dimensional perturbations, it is useful to adopt the spatial Fourier transform in the $y$ and $z$ directions;

$$
\boldsymbol{\xi}(x, y, z, t)=\frac{1}{2 \pi} \iint \check{\boldsymbol{\xi}}\left(x, k_{y}, k_{z}, t\right) e^{i\left(k_{y} y+k_{z} z\right)} \mathrm{d} k_{y} \mathrm{~d} k_{z} .
$$

Our task is to find the $(x, t)$-dependences of $\check{\boldsymbol{\xi}}\left(x, k_{y}, k_{z}, t\right)$ for fixed wavenumbers $k_{y}$ and $k_{z}$. To simplify the notations, we will denote this $\check{\boldsymbol{\xi}}\left(x, k_{y}, k_{z}, t\right)$ by $\boldsymbol{\xi}(x, t)$, omitting the check $\left(^{\sim}\right)$ and the $\left(k_{y}, k_{z}\right)$-dependences, which does not cause confusion in many cases. With respect to the fixed wavenumber vector $\boldsymbol{k}:=\left(0, k_{y}, k_{z}\right)$, we introduce the parallel and perpendicular components of $\boldsymbol{\xi}$ as follows.

$$
\xi_{\|}:=\frac{\boldsymbol{k} \cdot \boldsymbol{\xi}}{k}, \quad \xi_{\perp}:=\frac{\left(\boldsymbol{e}_{x} \times \boldsymbol{k}\right) \cdot \boldsymbol{\xi}}{k},
$$

where $k=|\boldsymbol{k}|$ and $\boldsymbol{e}_{x}=(1,0,0)$. In this manner, we shall use $(x, \|, \perp)$ components rather than $(x, y, z)$.

Let $\boldsymbol{\Xi}(x, \Omega)$ be the Laplace transform of $\boldsymbol{\xi}(x, t)$ again. We can algebraically eliminate $\Xi_{\|}$and $\Xi_{\perp}$ from (49) after the spatial Fourier transformation. As is shown generally in Appendix $\mathrm{C}$, such the elimination of variables always results in a new symmetric response equation;

$$
\mathcal{E}_{\mathrm{I}}(\Omega) \Xi_{x}(\Omega)=-\mathrm{m}_{0 \mathrm{I}}(\Omega),
$$

with the property $\mathcal{E}_{\mathrm{I}}^{*}(\Omega)=\mathcal{E}_{\mathrm{I}}(\bar{\Omega})$ in terms of the one-dimensional inner product $\int_{x_{1}}^{x_{2}} \bar{\circ} \circ \mathrm{d} x$. The left hand side of this equation corresponds to the well-known eigenvalue problem of the Sturm-Liouville type

$$
\mathcal{E}_{\mathrm{I}} \Xi_{x}:=\rho \Pi_{A} \Xi_{x}+\partial_{x}\left[\rho \frac{\Pi_{S} \Pi_{A}}{\Pi_{s f}} \partial_{x} \Xi_{x}\right]
$$

which was derived by Hain and Lüst [21], and Goedbloed [22] for the case of static equilibrium [but the generalization to the steady equilibrium (60) is straightforward]. The right hand side of (64) represents the initial data which can be computed as

$$
\mathrm{m}_{0 \mathrm{I}}:=\mathrm{m}_{0 x}-i k\left[\frac{\left(\Pi_{S}-\Pi_{v} b_{\|}^{2}\right) \mathrm{m}_{0 \|}-\Pi_{v} b_{\|} b_{\perp} \mathrm{m}_{0 \perp}}{\Pi_{s f}}\right]^{\prime} .
$$

In the above expressions, we defined some functions of $x$ and $\Omega$ as follows.

$$
\begin{aligned}
\Pi_{v}(x, \Omega) & =\left[\Omega-k v_{\|}(x)\right]^{2}, \\
\Pi_{A}(x, \Omega) & =\Pi_{v}(x, \Omega)-\omega_{A}^{2}(x), \\
\Pi_{S}(x, \Omega) & =\left[b^{2}(x)+c_{s}^{2}(x)\right]\left[\Pi_{v}(x, \Omega)-\omega_{S}^{2}(x)\right], \\
\Pi_{s f}(x, \Omega) & =\Pi_{v}^{2}(x, \Omega)-k^{2} \Pi_{S}(x, \Omega) \\
& =\left[\Pi_{v}(x, \Omega)-\omega_{s}^{2}(x)\right]\left[\Pi_{v}(x, \Omega)-\omega_{f}^{2}(x)\right],
\end{aligned}
$$


where $\boldsymbol{b}=\boldsymbol{B} / \sqrt{\rho}$ denotes the Alfvén speed and $c_{s}=\sqrt{\partial p(\rho, s) /\left.\partial \rho\right|_{s}}$ the sound speed. The following characteristic frequencies are conventional,

$$
\begin{gathered}
\omega_{A}^{2}=k^{2} b_{\|}^{2}: \text { Alfvén frequency, } \\
\omega_{S}^{2}=k^{2} \frac{b_{\|}^{2} c_{s}^{2}}{b^{2}+c_{s}^{2}}: \text { slow magneto-sonic frequency, } \\
\omega_{s, f}^{2}=\frac{k^{2}}{2}\left[\left(b^{2}+c_{s}^{2}\right) \pm \sqrt{\left(b^{2}+c_{s}^{2}\right)^{2}-4 b_{\|}^{2} c_{s}^{2}}\right] \\
: \text { slow }(-) \text { and fast }(+) \text { turning point frequencies. }
\end{gathered}
$$

The functions $\Pi_{A}, \Pi_{S}$ and $\Pi_{s f}$ may vanish when $\Pi_{v}$ is equal to the square of these frequencies. The set of $\omega \in \mathbb{R}$ for which there exists $x_{0} \in\left[x_{1}, x_{2}\right]$ satisfying either $\Pi_{A}\left(x_{0}, \omega\right)=0$ or $\Pi_{S}\left(x_{0}, \omega\right)=0$ corresponds to the continuous spectrum because such a point $x_{0}$ is a regular singular point of the ordinary differential equation (64). On the other hand, the singular point $x_{0}$ satisfying $\Pi_{s f}\left(x_{0}, \omega\right)=0$ is known to be apparent [41], that is, the solution $\Xi_{x}(x, \Omega)$ remains regular at such $\left(x_{0}, \omega\right)$. The continuous spectrum $\sigma_{c}$ consists of

$$
\begin{aligned}
\text { Alfvén continuous spectrum } & \sigma_{A}=\left\{k v_{\|}(x) \pm \omega_{A}(x) \in \mathbb{R}: x \in\left[x_{1}, x_{2}\right]\right\}, \\
\text { Slow continuous spectrum } & \sigma_{S}=\left\{k v_{\|}(x) \pm \omega_{S}(x) \in \mathbb{R}: x \in\left[x_{1}, x_{2}\right]\right\},
\end{aligned}
$$

which may overlap each other and may fold by itself depending on the profiles of the mean fields and the wave number $\boldsymbol{k}$. Moreover, if either $b_{\|}(x)$ or $b_{\perp}(x)$ vanishes somewhere in $\left[x_{1}, x_{2}\right]$, some frequencies out of $\omega_{A}(x), \omega_{S}(x), \omega_{s}(x)$ and $\omega_{f}(x)$ would degenerate and yield a different type of singularity in the equation [even in the incompressible case, these continuous spectra exhibit a nontrivial singularity [32] when $\left.b_{\|}(x)=0\right]$. We are planning detailed and systematic investigation of the MHD continuous spectrum in an upcoming paper, accompanied with some physical considerations. In this paper, we focus on the typical case, $b_{\|}(x) \neq 0$ and $b_{\perp}(x) \neq 0$ for all $x$, where the above four frequencies are separated.

Again, let us define the generalized dispersion relation for (64) by

$$
D_{\mathrm{I}}\left(\Omega, \xi_{x 1}, \xi_{x 2}\right)=\int_{x_{1}}^{x_{2}} \overline{\xi_{x 1}} \mathcal{E}_{\mathrm{I}}(\Omega) \xi_{x 2} \mathrm{~d} x \quad \text { for } \forall \xi_{x 1}, \xi_{x 2} .
$$

The following theorem holds in the same manner as Theorem 7 .

Theorem 8. For the slab MHD equilibria, the wave action for a semi-simple point spectrum $\omega_{j} \in \sigma_{p}$ is given by

$$
\begin{aligned}
\mu_{p}\left(\omega_{j}\right) & =i \int_{x_{1}}^{x_{2}} \overline{\hat{\xi}_{x}\left(\overline{\omega_{j}}\right)} \mathrm{m}_{0 \mathrm{I}}\left(\omega_{j}\right) \mathrm{d} x \\
& =\frac{\partial D_{\mathrm{I}}}{\partial \Omega}\left(\omega_{j}, \hat{\xi}_{x}\left(\overline{\omega_{j}}\right), \hat{\xi}_{x}\left(\omega_{j}\right)\right) \\
& =\int_{x_{1}}^{x_{2}} 2 \rho\left(\omega_{j}-k v_{\|}\right)\left[\overline{\hat{\xi}_{x}\left(\bar{\omega}_{j}\right)} \hat{\xi}_{x}\left(\omega_{j}\right)+k^{2} N\left(x, \omega_{j}\right) \overline{\partial_{x} \hat{\xi}_{x}\left(\bar{\omega}_{j}\right)} \partial_{x} \hat{\xi}_{x}\left(\omega_{j}\right)\right] \mathrm{d} x,
\end{aligned}
$$

where

$$
N\left(x, \omega_{j}\right)=\left.\frac{b_{\|}^{2} b_{\perp}^{2} \Pi_{v}^{2}+\left(\Pi_{S}-\Pi_{v} b_{\|}^{2}\right)^{2}}{\Pi_{s f}^{2}}\right|_{\Omega=\omega_{j}}
$$


If $b_{\|}(x) \neq 0$ and $b_{\perp}(x) \neq 0$ for all $x$, the wave action for continuous spectrum $\omega \in \sigma_{c}$ is given by

$$
\mu_{c}(\omega)=i \int_{x_{1}}^{x_{2}} \overline{\hat{\xi}_{x}(\omega)} \mathrm{m}_{0 \mathrm{I}}(\omega) \mathrm{d} x .
$$

Proof. The elimination of variables is discussed in Appendix $\mathrm{C}$ in a general manner. One can identify (49), being Fourier-transformed in $y$ and $z$ directions, as $(\mathrm{C} 7)$ by the correspondences $\mathbf{Z}_{a}=\left(\Xi_{x}\right)$ and $Z_{b}=\left(\Xi_{\|}, \Xi_{\perp}\right)^{T}$. After the elimination, the equation (64) parallels (C10). The dispersion relation $D_{\mathrm{I}}$ is then related to $D_{\mathrm{FR}}$ through the formula (C14), in which the term $\left\langle\overline{\chi_{b}}, \mathcal{E}_{b b}^{-1} \chi_{b}\right\rangle_{b}$ is easily computed as

$$
\int_{x_{1}}^{x_{2}} \overline{\left.\overline{\left(\mathrm{m}_{0 \|}\right.} \mathrm{m}_{0 \perp}\right)} \cdot \frac{1}{\rho \Pi_{s f}}\left(\begin{array}{cc}
\Pi_{A} & -k^{2} b_{\|} b_{\perp} \\
-k^{2} b_{\|} b_{\perp} & \Pi_{v}-k^{2}\left(b_{\perp}^{2}+c_{s}^{2}\right)
\end{array}\right)\left(\begin{array}{c}
\mathrm{m}_{0 \|} \\
\mathrm{m}_{0 \perp}
\end{array}\right) \mathrm{d} x .
$$

This expression is regular in terms of $\Omega$ except for the apparent singularities stemming from $\Pi_{s f}=0$. Since the apparent singularities are isolated from the genuine singularities, $\Pi_{A}=0$ and $\Pi_{S}=0$, due to the assumption $b_{\|}, b_{\perp} \neq 0$, we can use $D_{\text {I }}$ instead of $D_{\text {FR }}$ (and also $D$ ) when calculating the wave actions by (41) and (42). The remaining part of the proof is the same as Theorem 7 . The computation of the $\Omega$-derivative in (75) results in (76).

For a real point spectrum $\omega_{j} \in \mathbb{R}$, the integrand of (76) is positive except for $\omega_{j}-k v_{\|}(x)$. It follows that the wave action is positive (respectively, negative) if the phase velocity $\omega_{j} / k$ of the wave is faster (respectively, slower) than $v_{\|}(x)$, i.e. the velocity of the basic flow along the $\boldsymbol{k}$ direction, everywhere on $\left[x_{1}, x_{2}\right]$. When the Hopf bifurcation is concerned, linear instability occurs through the Krein collision (namely, coalescing on the real axis and splitting toward the upper and lower half planes) between a pair of eigenvalues with positive and negative actions. Such the collision is possible only when $\omega_{j}$ is included in the region $\left\{k v_{\|}(x) \in \mathbb{R}: x \in\left[x_{1}, x_{2}\right]\right\}$, from which any unstable eigenvalue $\Im\left(\omega_{j}\right)>0$ must emerge.

However, we must recall that there exists the continuous spectrum on the real axis. It seems that, besides the conventional Krein collision, degeneracy between point and continuous spectra or one between two continua may cause linear instability as was pointed out by Balmforth and Morrison [15]. To derive the sign of $\mu_{c}(\omega)$ requires some careful treatment of the singularity. We demonstrate this technique for the hydrodynamic case in the next section. Our result includes that of Ref. 15 and the derivation becomes more straightforward by means of the hyperfunction theory. The similar technique probably applies to the MHD continuous spectrum, which will be reported in the upcoming paper.

\section{PARALLEL SHEAR FLOW}

We can recover the hydrodynamic case easily by putting $\boldsymbol{B}=0$ in the MHD case. The equation $\mathcal{E}_{\mathrm{I}}(\Omega) \Xi_{x}(\Omega)=-\mathrm{m}_{0 \mathrm{I}}(\Omega)$ that was given in (64) reduces to

$$
\mathcal{E}_{\mathrm{I}} \Xi_{x}=\rho \Pi_{v} \Xi_{x}+\partial_{x}\left[\frac{\rho c_{s}^{2} \Pi_{v}}{\Pi_{v}-k^{2} c_{s}^{2}} \partial_{x} \Xi_{x}\right]
$$


and

$$
\begin{aligned}
\mathrm{m}_{0 \mathrm{I}} & =\mathrm{m}_{0 x}-i k\left[\frac{c_{s}^{2} \mathrm{~m}_{0 \|}}{\Pi_{v}-k^{2} c_{s}^{2}}\right]^{\prime} \\
& =-i \rho\left(\Omega-k v_{\|}\right) \xi_{0 x}-\left(\rho \boldsymbol{v} \cdot \boldsymbol{\xi}_{0}^{\prime}+\rho \alpha_{0}^{\prime}-\beta_{0} s^{\prime}\right)-k\left[\frac{\rho c_{s}^{2}\left(\Omega \xi_{0 \|}+k v_{\perp} \xi_{0 \perp}+k \alpha_{0}\right)}{\Pi_{v}-k^{2} c_{s}^{2}}\right]^{\prime}
\end{aligned}
$$

The singularity caused by $\Pi_{v}-k^{2} c_{s}^{2}=0$ in this equation is again apparent. Notice that both the Alfvén and slow continuous spectra degenerate into

$$
\sigma_{c}=\left\{k v_{\|}(x) \in \mathbb{R}: x \in\left[x_{1}, x_{2}\right]\right\},
$$

in the hydrodynamic case and the genuine singularity is caused only by $\Pi_{v}=0$. The theorem 8 holds in the limit of $\boldsymbol{B}=0$, where the function $N\left(x, \omega_{j}\right)$ is simplified to

$$
N\left(x, \omega_{j}\right)=\frac{c_{s}^{4}}{\left[\left(\omega_{j}-k v_{\|}\right)^{2}-k^{2} c_{s}^{2}\right]^{2}} .
$$

The proof, however, needs a little care since the expression (79) now becomes

$$
\left.\int_{x_{1}}^{x_{2}} \overline{\left(\mathrm{m}_{0 \|}\right.} \mathrm{m}_{0 \perp}\right) \cdot \frac{1}{\rho}\left(\begin{array}{cc}
\frac{1}{\Pi_{v}-k^{2} c_{s}^{2}} & 0 \\
0 & \frac{1}{\Pi_{v}}
\end{array}\right)\left(\begin{array}{l}
\mathrm{m}_{0 \|} \\
\mathrm{m}_{0 \perp}
\end{array}\right) \mathrm{d} x .
$$

and the singularity of the term $1 / \Pi_{v}$ coincides with the continuous spectrum $\sigma_{c}$. Nevertheless, we find that this expression is still regular with respect to $\Omega$ (except for the apparent singularity), by noting the fact that the expression $\mathrm{m}_{0 \perp}(\Omega)=-i \rho\left(\Omega-k v_{\|}\right) \xi_{0 \perp}$ is proportional to $\left(\Omega-k v_{\|}\right)$and the singularity $1 / \Pi_{v}$ in $(85)$ will be always canceled out.

Now, let us seek another expression of $\mu_{c}(\omega)$ that would be more useful than (78) for discussing its sign. The result hinges on the suitable transformation of variable. We introduce a new variable $\Phi(\Omega)$ as

$$
\Xi_{x}(\Omega)=\frac{\Phi(\Omega)}{\Omega-k v_{\|}(x)}
$$

In Appendix $\mathrm{C}$, we also show how this kind of transformation acts on the equation. Since the transformation operator $\mathcal{T}(\Omega)=\left[\Omega-k v_{\|}(x)\right]^{-1}$ satisfies the condition $\mathcal{T}^{*}(\Omega)=\mathcal{T}(\bar{\Omega})$, it necessarily produces a new symmetric response equation;

$$
\mathcal{E}_{\mathrm{II}}(\Omega) \Phi(\Omega)=-\mathrm{m}_{0 \mathrm{II}}(\Omega),
$$

where

$$
\mathcal{E}_{\mathrm{II}} \Phi:=\left[\rho+\frac{c_{s}^{2}\left(\rho k v_{\|}^{\prime}\right)^{\prime}}{\left(\Pi_{v}-k^{2} c_{s}^{2}\right)\left(\Omega-k v_{\|}\right)}+\rho c_{s} \frac{2 c_{s}\left(k v_{\|}^{\prime}\right)^{2}-c_{s}^{\prime} \Pi_{v}^{\prime}}{\left(\Pi_{v}-k^{2} c_{s}^{2}\right)^{2}}\right] \Phi+\partial_{x}\left[\frac{\rho c_{s}^{2}}{\Pi_{v}-k^{2} c_{s}^{2}} \partial_{x} \Phi\right]
$$

and

$$
\mathrm{m}_{0 I \mathrm{II}}:=\frac{\mathrm{m}_{0 \mathrm{I}}}{\Omega-k v_{\|}}
$$


Although this equation looks more formidable than its original form, the purpose of the variable transformation is to regularize the singularity of $\Xi_{x}(\Omega)$ appropriately. For a given $\omega \in \sigma_{c}$, there is (at least) one regular singular point $x=x_{c}$ such that $\omega-k v_{\|}\left(x_{c}\right)=0$, which is so-called the critical layer. Recall that the behavior of the solution in the neighborhood of $x_{c}$ is obtained by the Frobenius method of expansion $[42,43]$ and, thereby, $\Xi_{x}(x, \Omega)$ is found to be a linear combination of two independent solutions, say,

$$
\begin{aligned}
& \xi_{1}(x, \Omega)=A_{0}+A_{1} s+A_{2} s^{2}+\ldots, \\
& \xi_{2}(x, \Omega)=\xi_{1}(x, \Omega) \ln s+B_{0} s^{-1}+B_{1}+B_{2} s+\ldots
\end{aligned}
$$

where $s \in \mathbb{C}$ is the complex continuation of the real variable $x-x_{c}$; we have $\Re[s]=x-x_{c}$ and $\Omega-k v_{\|}(x)=-k v_{\|}^{\prime}\left(x_{c}\right) s+O\left(s^{2}\right)$. Except for some special cases (such as $\left.v_{\|}^{\prime}\left(x_{c}\right)=0\right), \Xi_{x}(x, \Omega$ ) includes the term $1 / s$, which implies that the singular eigenfunction $\hat{\xi}_{x}(x, \omega)$ includes the delta function $\delta\left(\omega-k v_{\|}(x)\right)$ and the principal value of $1 /\left(\omega-k v_{\|}(x)\right)$. We have therefore transformed $\Xi_{x}$ to $\Phi$ so that this singularity $1 / s$ be regularized. It is interesting to note that this idea is analogous to (58) for point spectra. Since the singularity of $\Phi$ is at worst $s \ln s$, the generalized eigenfunction $\hat{\phi}$ (given below) is a continuous function.

For this variable transformation, the formula (C3) in Appendix C is helpful and the wave action can be represented in terms of $\hat{\phi}$ as follows.

Theorem 9. The wave action for the continuous spectrum $\sigma_{c}=\left\{k v_{\|}(x)\right\}$ is represented by

$$
\begin{aligned}
\mu_{c}(\omega)= & \frac{\pi^{2}}{k} \int_{x_{1}}^{x_{2}}\left(\rho v_{\|}^{\prime}\right)^{\prime} \delta\left(\omega-k v_{\|}\right)\left[|\hat{\phi}(\omega)|^{2}+\left|\hat{\phi}^{\dagger}(\omega)\right|^{2}\right] \mathrm{d} x \\
& -2 \pi \int_{x_{1}}^{x_{2}} \delta\left(\omega-k v_{\|}\right) \Re\left\{\overline{\hat{\phi}^{\dagger}(\omega)}\left[\left(\rho v_{\perp}\right)^{\prime} \xi_{0 \perp}+\rho^{\prime}\left(v_{\|} \xi_{0 \|}+\alpha_{0}\right)+s^{\prime} \beta_{0}\right]\right\} \mathrm{d} x,
\end{aligned}
$$

where the singular eigenfunction $\hat{\phi}(\omega)$ and its Hilbert transform $\hat{\phi}^{\dagger}(\omega)$ are, respectively, given by

$$
\begin{aligned}
\hat{\phi}(\omega) & =\frac{1}{2 \pi}[\Phi(\omega+i 0)-\Phi(\omega-i 0)], \\
\hat{\phi}^{\dagger}(\omega) & =\frac{1}{2 \pi}[\Phi(\omega+i 0)+\Phi(\omega-i 0)] .
\end{aligned}
$$

Proof. Note that the right most term of the formula (C14) will not be affected whenever we apply any variable transformation $\mathcal{T}(\Omega)$ satisfying $\mathcal{T}^{*}(\Omega)=\mathcal{T}(\bar{\Omega})$. Therefore, as we have done repeatedly in this work, we may use a new generalized dispersion relation,

$$
D_{\mathrm{II}}\left(\Omega, \phi_{1}, \phi_{2}\right)=\int_{x_{1}}^{x_{2}} \overline{\phi_{1}} \mathcal{E}_{\mathrm{II}}(\Omega) \phi_{2} \mathrm{~d} x,
$$

instead of $D$ in (42). Before taking the limit of $\Omega \rightarrow \omega \pm i 0$, we apply the formula (C3) to this dispersion relation $D_{\text {II }}$ and obtain

$$
\begin{aligned}
\mu_{c}(\omega)=\frac{1}{2 \pi i} \lim _{\Omega \rightarrow \omega+i 0} & \int_{x_{1}}^{x_{2}}\left[\overline{F(\Omega)}\left[\mathcal{E}_{\mathrm{II}}(\Omega)-\mathcal{E}_{\mathrm{II}}(\bar{\Omega})\right] F(\Omega)+\overline{G(\Omega)}\left[\mathcal{E}_{\mathrm{II}}(\Omega)-\mathcal{E}_{\mathrm{II}}(\bar{\Omega})\right] G(\Omega)\right. \\
& \left.+\overline{G(\Omega)}\left[\mathrm{m}_{0 \mathrm{II}}(\Omega)-\mathrm{m}_{0 \mathrm{II}}(\bar{\Omega})\right]+\overline{\left[\mathrm{m}_{0 \mathrm{II}}(\bar{\Omega})-\mathrm{m}_{0 \mathrm{II}}(\Omega)\right]} G(\Omega)\right] \mathrm{d} x,
\end{aligned}
$$


where

$$
\begin{aligned}
F(\Omega) & :=\frac{1}{2}[\Phi(\Omega)-\Phi(\bar{\Omega})], \\
G(\Omega) & :=\frac{1}{2}[\Phi(\Omega)+\Phi(\bar{\Omega})] .
\end{aligned}
$$

To estimate this limit, let us exploit the Plemelj formula (B7), which enables us to get

$$
\begin{aligned}
\mathcal{E}_{\mathrm{II}}(\omega+i 0)-\mathcal{E}_{\mathrm{II}}(\omega-i 0) & =2 \pi i \frac{\left(\rho v_{\|}^{\prime}\right)^{\prime}}{k} \delta\left(\omega-k v_{\|}\right), \\
\mathrm{m}_{0 \mathrm{II}}(\omega+i 0)-\mathrm{m}_{0 \mathrm{II}}(\omega-i 0) & =-2 \pi i \delta\left(\omega-k v_{\|}\right)\left[\left(\rho v_{\perp}\right)^{\prime} \xi_{0 \perp}+\rho^{\prime}\left(v_{\|} \xi_{0 \|}+\alpha_{0}\right)+s^{\prime} \beta_{0}\right] .
\end{aligned}
$$

By noting that $\pi \hat{\phi}(\omega)=F(\omega+i 0)$ and also $\pi \hat{\phi}^{\dagger}(\omega)=G(\omega+i 0)$ are continuous functions, the required result is immediately derived by substituting these expressions.

Since both the integrals in (92) include the delta function that may be rewritten as

$$
\delta\left(\omega-k v_{\|}(x)\right)=\frac{1}{\left|k v_{\|}^{\prime}\left(x_{c}\right)\right|} \delta\left(x-x_{c}\right)
$$

only the value of each integrand at $x=x_{c}$ is of interest to the calculation of $\mu_{c}(\omega)$. We, nevertheless, prefer the integral form (92) because of its simplicity and generality. For instance, the critical layer $x_{c}$ is not always unique for each $\omega \in \sigma_{c}$ when the shear profile $v_{\|}(x)$ is a non-monotonic function. The formula (92), without modification, can deal with such cases of several critical layers.

We remark that our result corresponds to that of Balmforth and Morrison [15] by restricting our problem to the incompressible 2D Euler equation, namely, by considering unidirectional parallel flows

$$
\boldsymbol{v}=\left(0, v_{y}(x), 0\right), \quad \rho(x)=\text { const. } \quad \text { and } \quad s(x)=\text { const. }
$$

and two-dimensional perturbations, $k_{z}=0$ and $\xi_{0 \perp} \equiv 0$. The incompressible limit $\left(c_{s} \rightarrow \infty\right)$ can be taken in the equation (87) and the eigenvalue problem $\mathcal{E}_{\mathrm{II}}(\omega) \hat{\phi}(\omega)=0$ corresponds to the Rayleigh equation [44]. In this case, the second integral in the right hand side of (92) vanishes and the sign of $\mu_{c}(\omega)$ is determined solely by that of $v_{y}^{\prime \prime}\left(x_{c}\right)$. As was pointed out in Ref. 15, this result agrees with the conventional stability theories such as Rayleigh's inflection theorem [44] and Arnold's energy criterion [1].

For compressible and fully three-dimensional perturbations, the sign of $\mu_{c}(\omega)$ is not so self-evident. We notice that this difficulty is attributed to the degeneracy of two continuous spectra on $\sigma_{c}$. Recall that there were two kinds of continuous spectrum, $\sigma_{A}$ and $\sigma_{S}$, in the MHD case and they are now exactly overlapping due to the limit $\boldsymbol{B} \rightarrow 0$. We can indirectly ensure this fact by choosing a certain initial data. For $\left(\boldsymbol{\xi}_{0}, \alpha_{0}, \beta_{0}\right)$ satisfying

$$
\left(\rho v_{\perp}\right)^{\prime} \xi_{0 \perp}+\rho^{\prime}\left(v_{\|} \xi_{0 \|}+\alpha_{0}\right)+s^{\prime} \beta_{0}=0,
$$

the wave action for $\omega \in \sigma_{c}$ becomes

$$
\mu_{c}(\omega)=\frac{\pi^{2}}{k} \int_{x_{1}}^{x_{2}}\left(\rho v_{\|}^{\prime}\right)^{\prime} \delta\left(\omega-k v_{\|}\right)\left[|\hat{\phi}(\omega)|^{2}+\left|\hat{\phi}^{\dagger}(\omega)\right|^{2}\right] \mathrm{d} x
$$


and the sign of $\mu_{c}(\omega)$ coincides with that of $\left(\rho v_{\|}^{\prime}\right)^{\prime}$ at $x=x_{c}$ (i.e., $\operatorname{sgn}\left[\mu_{c}(\omega)\right]=\operatorname{sgn}\left[\left(\rho v_{\|}^{\prime}\right)^{\prime}\left(x_{c}\right)\right]$ ) in the same way as the $2 \mathrm{D}$ Euler equation. On the other hand, for $\left(\boldsymbol{\xi}_{0}, \alpha_{0}, \beta_{0}\right)$ satisfying

$$
\left\{\begin{array}{l}
\xi_{0 x}^{\prime}+i k \xi_{0 \|}=0 \\
v_{\|}^{\prime} \xi_{0 x}+i k\left(\boldsymbol{v} \cdot \boldsymbol{\xi}_{0}+\alpha_{0}\right)=0, \\
\rho v_{\|}^{\prime \prime} \xi_{0 x}+i k\left(\rho v_{\perp}^{\prime} \xi_{0 \perp}+s^{\prime} \beta_{0}\right)=0
\end{array}\right.
$$

we can prove, after some manipulations, that $\Phi(x, \Omega)=i \xi_{0 x}(x)$ is the exact solution of $(87)$. The wave action (92), then, becomes

$$
\mu_{c}(\omega)=-\frac{1}{k} \int_{x_{1}}^{x_{2}}\left(\rho v_{\|}^{\prime}\right)^{\prime} \delta\left(\omega-k v_{\|}\right)\left|\xi_{0 x}\right|^{2} \mathrm{~d} x
$$

whose sign is exactly opposite of the previous case (104). This another kind of a continuous spectrum is provided by the (inviscid) Squire equation [43] for three-dimensional perturbations. With this example, we have illustrated that the wave action for each $\omega \in \sigma_{c}$ can be positive or negative depending on the initial data, which implies that there exist two overlapping continuous spectra associated with the positive and negative wave actions. Therefore, the energy of three-dimensional perturbation is by no means definite and, moreover, the coalescence between spectra of positive and negative energy modes (like the marginally stable state of the Krein collision) is realized everywhere on the two continuous spectra. While Krein's bifurcation theory is not yet established for a continuous spectrum, it is highly possible that the two continuous spectra are marginally stable, because some external forces or the deviation from the slab symmetry are known to cause three-dimensional local instabilities (see the section II.5.G. of Ref. 2 and references therein).

Finally, we extend the Krein's idea to the case of coalescence between point and continuous spectra. The following argument is traced back to the findings of Briggs et al. [25], though they were not aware of the viewpoint of energetics. We put emphasis on the appearance of the operator $\mathcal{E}_{\mathrm{II}}(\omega+i 0)-\mathcal{E}_{\mathrm{II}}(\omega-i 0)$ in the expression of $\mu_{c}(\omega)$ because it has implications of possible bifurcation as follows.

Let $\omega_{0} \in \mathbb{R}$ be a real point spectrum isolated from (but, close to) the continuous spectrum $\sigma_{c}=\left\{k v_{\|}(x)\right\}$ as illustrated in Fig. 2a. The corresponding eigenfunction $\hat{\phi}\left(\omega_{0}\right)$ satisfies the eigenvalue problem $\mathcal{E}_{\mathrm{II}}\left(\omega_{0}\right) \hat{\phi}\left(\omega_{0}\right)=0$ and, of course, the dispersion relation,

$$
D_{\mathrm{II}}\left(\omega_{0}, \hat{\phi}\left(\omega_{0}\right), \hat{\phi}\left(\omega_{0}\right)\right)=\int_{x_{1}}^{x_{2}} \overline{\hat{\phi}\left(\omega_{0}\right)} \mathcal{E}_{\mathrm{II}}\left(\omega_{0}\right) \hat{\phi}\left(\omega_{0}\right) \mathrm{d} x=0 .
$$

In the present case, such the point spectrum can certainly exists as the acoustic wave. If $\omega_{0}$ is on the right side of $\sigma_{c}$ as in Fig. 2a, its wave action $\mu_{p}\left(\omega_{0}\right)$ must be positive as was proved generally in Theorem 8 .

As is common in the bifurcation theory, we consider what happens when the mean flow profile slightly changes from $v_{\|}(x)$ to $v_{\|}(x)+\delta v_{\|}(x)$ such that $\omega_{0}$ enters the range of the new continuous spectrum $\sigma_{c}=\left\{k v_{\|}(x)+k \delta v_{\|}(x)\right\}$. The operator $\mathcal{E}_{\mathrm{II}}(\Omega)$, then, turns into $\mathcal{E}_{\mathrm{II}}(\Omega)+\delta \mathcal{E}_{\mathrm{II}}(\Omega)$, where $\delta \mathcal{E}_{\mathrm{II}}(\Omega)$ would be no longer regular at $\Omega=\omega_{0}$ due to $\omega_{0} \in \sigma_{c}$.

Assume that the point spectrum $\omega_{0}$ moves to $\omega_{0}+\delta \omega$ after the change. When $\delta \omega$ is complex, the other point spectrum $\omega_{0}+\overline{\delta \omega}$ must also exist and, hence, one may assume $\Im[\delta \omega] \geq 0$ without loss of generality. The eigenfunction $\hat{\phi}\left(\omega_{0}\right)$ would slightly change into

$$
\begin{array}{ll}
\hat{\phi}\left(\omega_{0}\right)+\delta \hat{\phi}_{+} & \text {for } \omega_{0}+\delta \omega \in \sigma_{p}, \\
\hat{\phi}\left(\omega_{0}\right)+\delta \hat{\phi}_{-} & \text {for } \omega_{0}+\overline{\delta \omega} \in \sigma_{p},
\end{array}
$$


a)

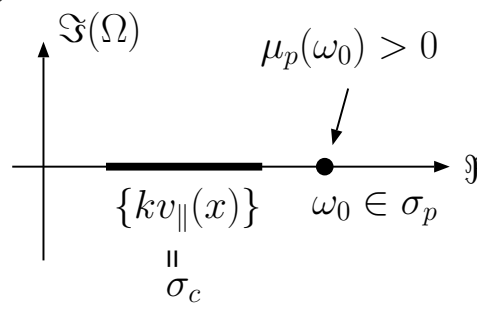

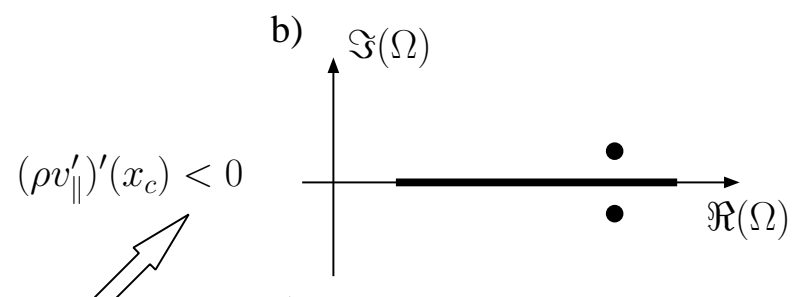

c)

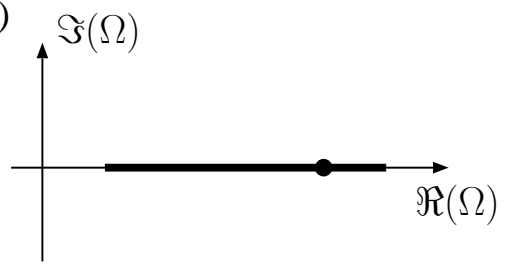

$$
\left(\rho v_{\|}^{\prime}\right)^{\prime}\left(x_{c}\right)>0
$$

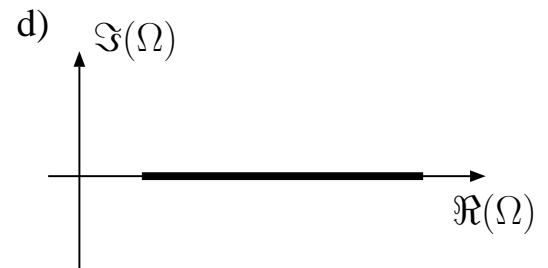

FIG. 2: Coalescence between point and continuous spectra

in such a way that the dispersion relation again holds. As for $\omega_{0}+\delta \omega \in \sigma_{p}$, it reads

$$
\int_{x_{1}}^{x_{2}} \overline{\left[\hat{\phi}\left(\omega_{0}\right)+\delta \hat{\phi}_{-}\right]}\left[\mathcal{E}_{\mathrm{II}}\left(\omega_{0}+\delta \omega\right)+\delta \mathcal{E}_{\mathrm{II}}\left(\omega_{0}+\delta \omega\right)\right]\left[\hat{\phi}\left(\omega_{0}\right)+\delta \hat{\phi}_{+}\right] \mathrm{d} x=0 .
$$

The variations $\delta \omega$ and $\delta \phi_{ \pm}$are supposed to be small. We carry out the Taylor expansion as follows,

$$
\mathcal{E}_{\mathrm{II}}\left(\omega_{0}+\delta \omega\right)+\delta \mathcal{E}_{\mathrm{II}}\left(\omega_{0}+\delta \omega\right)=\mathcal{E}_{\mathrm{II}}\left(\omega_{0}\right)+\frac{\partial \mathcal{E}_{\mathrm{II}}}{\partial \Omega}\left(\omega_{0}\right) \delta \omega+\delta \mathcal{E}_{\mathrm{II}}\left(\omega_{0}+i 0\right)+\ldots
$$

where the fact $\Im[\delta \omega] \geq 0$ has been used. At the first order, the dispersion relation can be written as

$$
\int_{x_{1}}^{x_{2}} \overline{\hat{\phi}\left(\omega_{0}\right)}\left[\frac{\partial \mathcal{E}_{\mathrm{II}}}{\partial \Omega}\left(\omega_{0}\right) \delta \omega+\delta \mathcal{E}_{\mathrm{II}}\left(\omega_{0}+i 0\right)\right] \hat{\phi}\left(\omega_{0}\right) \mathrm{d} x=0 .
$$

The same argument for the other point spectrum $\omega_{0}+\overline{\delta \omega} \in \sigma_{p}$ yields

$$
\int_{x_{1}}^{x_{2}} \overline{\hat{\phi}\left(\omega_{0}\right)}\left[\frac{\partial \mathcal{E}_{\mathrm{II}}}{\partial \Omega}\left(\omega_{0}\right) \overline{\delta \omega}+\delta \mathcal{E}_{\mathrm{II}}\left(\omega_{0}-i 0\right)\right] \hat{\phi}\left(\omega_{0}\right) \mathrm{d} x=0 .
$$

By taking the difference of these expressions, we can estimate the imaginary part of $\delta \omega$ as follows.

$$
\begin{aligned}
\Im[\delta \omega] & =-\frac{\frac{1}{2 i} \int_{x_{1}}^{x_{2}} \overline{\hat{\phi}\left(\omega_{0}\right)}\left[\delta \mathcal{E}_{\mathrm{II}}\left(\omega_{0}+i 0\right)-\delta \mathcal{E}_{\mathrm{II}}\left(\omega_{0}-i 0\right)\right] \hat{\phi}\left(\omega_{0}\right) \mathrm{d} x}{\int_{x_{1}}^{x_{2}} \overline{\hat{\phi}\left(\omega_{0}\right)} \frac{\partial \mathcal{E}_{\mathrm{II}}}{\partial \Omega}\left(\omega_{0}\right) \hat{\phi}\left(\omega_{0}\right) \mathrm{d} x}, \\
& =-\frac{\frac{\pi}{k} \int_{x_{1}}^{x_{2}}\left(\rho v_{\|}^{\prime}\right)^{\prime} \delta\left(\omega_{0}-k v_{\|}\right)\left|\hat{\phi}\left(\omega_{0}\right)\right|^{2} \mathrm{~d} x}{\mu_{p}\left(\omega_{0}\right)},
\end{aligned}
$$


The denominator exactly corresponds to the wave action $\mu_{p}\left(\omega_{0}\right)$ that the point spectrum $\omega_{0} \in \sigma_{p}$ originally has. Since we know $\mu_{p}\left(\omega_{0}\right)>0$ in Fig. 2 , we can predict how this eigenvalue $\omega_{0}$ will deviate from the real axis by observing the sign of the numerator. Note that the numerator has a similar expression to the first term of $\mu_{c}\left(\omega_{0}\right)$ given by (92) (namely, the wave action of the continuous spectrum $\omega_{0} \in \sigma_{c}$ that appears after the change), because they are derived from the same limit $\mathcal{E}_{\mathrm{II}}(\omega+i 0)-\mathcal{E}_{\mathrm{II}}(\omega-i 0)$. While we found two kinds of continuous spectrum containing opposite signs of the wave actions (104) and (106), only the former one is related to the movement of the point spectrum (the latter one seems to be decoupled in this setting). Let $x=x_{c}$ be the critical layer corresponding to $\omega_{0} \in \sigma_{c}$. If this continuous spectrum has negative wave action $\left(\rho v_{\|}^{\prime}\right)^{\prime}\left(x_{c}\right)<0$, there will be two point spectra $\omega_{0}+\delta \omega$ and $\omega_{0}+\overline{\delta \omega}$ representing, respectively, exponentially growing and damping waves (Fig. $2 \mathrm{~b}$ ). Note that, apart from an infinite number of singular modes, these two modes originate from only one neutral mode that is free from the critical layer. This phenomenon, so-called the critical layer instability [23], was investigate by Iga [24] in a different configuration addressing the two-layer problem of a geostrophic flow, where he evaluated the pseudomomentum (or wave momentum) that corresponds to the wave action multiplied by the wave number in our context. Only when $\left(\rho v_{\|}^{\prime}\right)^{\prime}\left(x_{c}\right)=0$, the point spectrum will remain on the real axis and will be embedded in the continuous spectrum (Fig. 2c) [42].

It is remarkable that, if the continuous spectrum has positive wave action $\left(\rho v_{\|}^{\prime}\right)^{\prime}\left(x_{c}\right)>0$, the assumption $\Im(\delta \omega) \geq 0$ is violated, implying that the point spectrum will vanish from the complex plane (Fig. 2d). Even in this case, the expression (115) has a special meaning, namely, it indicates the Landau damping of the mode [25]. By the analytic continuation of $\Phi(\Omega)$ from the upper half plane into the lower one over the singularity of the continuous spectrum $\sigma_{c}$ [which is indeed a branch cut of $\Phi(\Omega)$ ], we can find a fictious point spectrum on another Riemannian sheet as shown in Fig. 3. Although the genuine spectrum is still $\sigma_{c} \subset \mathbb{R}$, the inverse Laplace transform admits such the equivalent deformation of singularities (from Fig. 2d to Fig. 3). Actually, the transient behavior stemming from $\sigma_{c}$ exhibits the damping mode predicted by (115),

$$
\int_{\sigma_{c}} \hat{\phi}(\omega) e^{-i \omega t} \mathrm{~d} \omega=\left[\hat{\phi}\left(\omega_{0}\right)+\delta \hat{\phi}_{+}\right] e^{-i\left(\omega_{0}+\delta \omega\right) t}+O\left(t^{-2}\right) .
$$

This exponential behavior, so-called the Landau damping, will be eventually overwhelmed by the long-term algebraic decay $O\left(t^{-2}\right)$ of the superposed singular modes [34, 45]. The Landau damping of a vortical mode in incompressible shear flows was found by Briggs et al. [25], and we saw that their result can be generalized to any neutral waves (including acoustic waves) in compressible shear flows. The most striking observation that we made here is the expression for the growth or damping rate $\Im[\delta \omega]$ in (114) whose denominator and numerator are, respectively, associated with the wave actions of the point and continuous spectra. It seems that the formula (114) applies to more general cases not only to parallel shear flows. Our demonstration suggests that the "Krein collision" between point and continuous spectra may be generally classified in the same way as Fig. 2 .

\section{SUMMARY}

In this work, we provide a solid definition of wave energy starting with the noncanonical Hamiltonian theory and applying the spectral analysis (or the Laplace transform) to the 


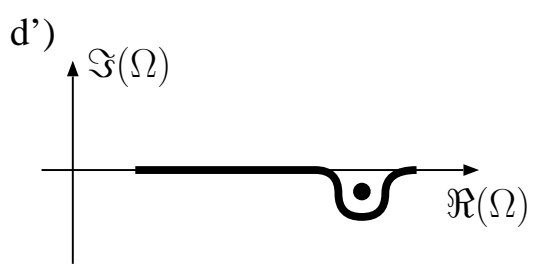

FIG. 3: Landau damping of the point spectrum

linearized system. We assumed in (A) that initial perturbations are kinematically accessible from the unperturbed state (i.e. the perturbed state is accessible without violating all kinematical conservation laws). Under this assumption, the linear perturbation $\tilde{u}(t)$ remains kinematically accessible for all $t>0$ and there is an adjoint variable $\zeta(t)$ which is combined with the linear solution through a mapping, $\tilde{u}(t)=\mathcal{A} \zeta(t)$. While the dynamical system is originally noncanonical, linear perturbations restricted in this way are endowed with many canonical features. The energy of perturbation can be defined by $\delta^{2} H=\left\langle\tilde{u}, \partial_{t} \zeta\right\rangle$, which is indeed a constant of motion. When the perturbation is decomposed into eigenmodes, the spectrum $\sigma \subset \mathbb{C}$ (i.e. complex frequencies of the eigenmodes) satisfies $\sigma=\bar{\sigma}=-\sigma=-\bar{\sigma}$ like canonical systems. In Theorem 5, the energy of each eigenmode is represented by the form of $($ energy $)=($ frequency $) \times($ action $)$ in an analogous manner to the simple harmonic oscillator. By generalizing the notion of dispersion relation appropriately, we could express the action variable, or the wave action, in terms of it. This expression takes a different appearance between for a point spectrum (41) and for a continuous spectrum (42).

Under the kinematical constraint (A), the linearized MHD equation reduces to the Frieman-Rotenberg (FR) equation, where we remarked that the initial data should be somewhat restricted as shown in (46). A generalized dispersion relation (53) was established for the FR equation on the ground of the general framework developed in Sec. III. By specializing the slab geometry of the equilibrium (60), the FR equation further reduced to the ordinary differential equation (64) of Sturm-Liouville type with a complicated inhomogeneous term. Even for this equation, the formulae (41) and (42) remains applicable in terms of the newly defined dispersion relation. At all events, the wave action corresponding to the point spectrum can be always represented by the derivative of the dispersion relation with respect to the frequency as exemplified by (55) and (75), which is a generalization of the conventional formula (2) for plane waves. The sign of the wave action can be read off if the phase velocity of the wave is faster (or slower) than the mean flow everywhere. Even for strongly inhomogeneous mean fields, our formula computes the wave action of each mode by substituting the eigenvalue and the eigenfunction.

On the contrary, the same argument is not true for a continuous spectrum and associated singular eigenmodes. To extract a satisfactory expression for the wave action from (42), the detailed information about the singularity is required for an appropriate choice of the variable transformation. Once we have worked it out, the use of the identity (C3) for the dispersion relation seems to be effective when estimating the limit $\Omega \rightarrow \omega \pm i 0$ of (42). For simplicity, we considered parallel shear flows with no magnetic field, where the regular singular point of the Sturm-Liouville equation yields the singular solution $\Xi_{x}(x, \Omega)$ containing $1 /\left[\Omega-k v_{\|}(x)\right]$. In this case, we chose the variable transformation (86) such that $\Phi(x, \Omega)$ will be continuous even when $\Omega \rightarrow \omega \pm i 0$. After this transformation, we could represent the wave action of each singular eigenmode by (92). Our result includes that of Ref. 15 and generalizes 
it to the case of compressible and three-dimensional perturbations. For three-dimensional perturbations, there are two kinds of continuous spectrum overlapping with each other. And, furthermore, the corresponding wave actions have opposite signs, which explains why the energy of perturbation $\left(\delta^{2} H\right)$ is hopelessly indefinite in the three-dimensional case as was observed in many literatures.

By taking advantage of this successful example, we examined the Krein collision between point and continuous spectra by adapting Briggs' approach. The formula (114), which seems to be universal, predicts the following bifurcation processes. If the signs of the wave actions for point and continuum were opposite, the single point spectrum would split into two ones which represent exponentially growing and damping waves. On the other hand, if the signs were same, the point spectrum would disappear from the complex plane, while the corresponding wave would be observed transiently as if a damping mode (the Landau damping). Our schematic picture Fig. 2 goes beyond the scope of the original Krein collision between two point spectra, though the bifurcation is inferred from the knowledge of the signs of actions. The generalization to the collision between two continua seems to be more involved but will be discussed elsewhere based on this work.

\section{APPENDIX A: LINEAR OPERATORS IN THE LINEARIZED MHD EQUATION}

The linearized MHD equation for perturbations $\tilde{u}=(\tilde{\boldsymbol{M}}, \tilde{\boldsymbol{B}}, \tilde{\rho}, \tilde{s})^{T}$ is represented by the form of (E), where the linear operators $\mathcal{A}, \mathcal{H}$ and $\mathcal{B}$ are explicitly written in $4 \times 4$ matrix forms as follows.

$$
\begin{gathered}
\mathcal{A}=\left(\begin{array}{cccc}
-\boldsymbol{M} \nabla \cdot \circ-\nabla(\boldsymbol{M} \cdot \circ)-(\nabla \times \boldsymbol{M}) \times \circ-\boldsymbol{B} \times(\nabla \times \circ) & -\rho \nabla \circ & \circ \nabla s \\
\nabla \times(\circ \times \boldsymbol{B}) & 0 & 0 & 0 \\
-\nabla \cdot(\rho \circ) & 0 & 0 & 0 \\
-\circ \cdot \nabla s & 0 & 0 & 0
\end{array}\right), \\
\mathcal{H}=\left(\begin{array}{cccc}
\frac{1}{\rho} & 0 & -\frac{\boldsymbol{M}}{\rho^{2}} & 0 \\
0 & 1 & 0 & 0 \\
-\frac{\boldsymbol{M}}{\rho^{2}} & 0 & \frac{|\boldsymbol{M}|^{2}}{\rho^{3}}+\frac{\partial^{2}(\rho e)}{\partial \rho^{2}} & \frac{\partial^{2}(\rho e)}{\partial \rho \partial} \\
0 & 0 & \frac{\partial^{2}(\rho e)}{\partial \rho \partial s} & \frac{\partial^{2}(\rho e)}{\partial s^{2}}
\end{array}\right), \\
\mathcal{B}=\left(\begin{array}{cccc}
-\nabla \cdot \boldsymbol{v}-\nabla(\circ \cdot \boldsymbol{v})-(\nabla \times \circ) \times \boldsymbol{v}(\nabla \times \boldsymbol{B}) \times \circ & \nabla\left[\frac{|\boldsymbol{v}|^{2}}{2}-h\right] & \rho T \nabla \circ \\
0 & \nabla \times(\boldsymbol{v} \times \circ) & 0 & 0 \\
0 & 0 & -\nabla \cdot(\circ \boldsymbol{v}) & 0 \\
0 & 0 & 0 & -\boldsymbol{v} \cdot \nabla \circ
\end{array}\right),
\end{gathered}
$$

where $h(\rho, s)=\partial[\rho e(\rho, s)] / \partial \rho$ denotes the enthalpy per unit mass and $T(\rho, s)=\partial e(\rho, s) / \partial s$ the temperature. One can derive these operators by linearizing the Lie-Poisson structure of the MHD equation [17, 20, 26].

\section{APPENDIX B: PROOF OF LEMMA 4}

Consider the Laplace transform of the two equations $\left(\mathrm{E}^{\prime}\right)$ and $\left(\mathrm{E}^{\prime *}\right)$;

$$
\begin{gathered}
(\Omega-\mathcal{L}) U(\Omega)=i \tilde{u}_{0}, \\
\left(\Omega^{\prime}-\mathcal{L}^{*}\right) \mathrm{Z}\left(\Omega^{\prime}\right)=i \zeta_{0} .
\end{gathered}
$$


The inner products, $\left\langle\overline{(B 1)}, \mathrm{Z}\left(\Omega^{\prime}\right)\right\rangle$ and $\langle\overline{U(\Omega)},(B 2)\rangle$, lead to

$$
\begin{aligned}
& \bar{\Omega}\left\langle\overline{U(\Omega)}, \mathrm{Z}\left(\Omega^{\prime}\right)\right\rangle-\left\langle\overline{\mathcal{L} U(\Omega)}, \mathrm{Z}\left(\Omega^{\prime}\right)\right\rangle=-i\left\langle\overline{\tilde{u}_{0}}, \mathrm{Z}\left(\Omega^{\prime}\right)\right\rangle, \\
& \Omega^{\prime}\left\langle\overline{U(\Omega)}, \mathrm{Z}\left(\Omega^{\prime}\right)\right\rangle-\left\langle\overline{\mathcal{L} U(\Omega)}, \mathrm{Z}\left(\Omega^{\prime}\right)\right\rangle=i\left\langle\overline{U(\Omega)}, \zeta_{0}\right\rangle .
\end{aligned}
$$

By taking the difference of these two expressions, we get

$$
\left(\Omega^{\prime}-\bar{\Omega}\right)\left\langle\overline{U(\Omega)}, \mathrm{Z}\left(\Omega^{\prime}\right)\right\rangle=i\left[\left\langle\overline{U(\Omega)}, \zeta_{0}\right\rangle+\left\langle\overline{\tilde{u}_{0}}, \mathrm{Z}\left(\Omega^{\prime}\right)\right\rangle\right]
$$

From the definitions (30) and (31), we can calculate the inner product in question as follows,

$$
\begin{aligned}
\left\langle\overline{\hat{\tilde{u}}(\omega)}, \hat{\zeta}\left(\omega^{\prime}\right)\right\rangle= & \lim _{\epsilon, \epsilon^{\prime} \rightarrow+0} \frac{1}{4 \pi^{2}}\left[\left\langle\overline{U(\omega+i \epsilon)}, \mathrm{Z}\left(\omega^{\prime}+i \epsilon^{\prime}\right)\right\rangle-\left\langle\overline{U(\omega+i \epsilon)}, \mathrm{Z}\left(\omega^{\prime}-i \epsilon^{\prime}\right)\right\rangle\right. \\
& \left.-\left\langle\overline{U(\omega-i \epsilon)}, \mathrm{Z}\left(\omega^{\prime}+i \epsilon^{\prime}\right)\right\rangle+\left\langle\overline{U(\omega-i \epsilon)}, \mathrm{Z}\left(\omega^{\prime}-i \epsilon^{\prime}\right)\right\rangle\right], \\
= & \lim _{\epsilon, \epsilon^{\prime} \rightarrow+0} \frac{i}{4 \pi^{2}}\left[\frac{\left\langle\overline{U(\omega+i \epsilon)}, \zeta_{0}\right\rangle+\left\langle\overline{\tilde{u}_{0}}, \mathrm{Z}\left(\omega^{\prime}+i \epsilon^{\prime}\right)\right\rangle}{-(\omega-i \epsilon)+\omega^{\prime}+i \epsilon^{\prime}}\right. \\
& -\frac{\left\langle\overline{U(\omega+i \epsilon)}, \zeta_{0}\right\rangle+\left\langle\overline{\tilde{u}_{0}}, \mathbf{Z}\left(\omega^{\prime}-i \epsilon^{\prime}\right)\right\rangle}{-(\omega-i \epsilon)+\omega^{\prime}-i \epsilon^{\prime}} \\
& -\frac{\left\langle\overline{U(\omega-i \epsilon)}, \zeta_{0}\right\rangle+\left\langle\overline{\tilde{u}_{0}}, \mathbf{Z}\left(\omega^{\prime}+i \epsilon^{\prime}\right)\right\rangle}{-(\omega+i \epsilon)+\omega^{\prime}+i \epsilon^{\prime}} \\
& \left.+\frac{\left\langle\overline{U(\omega-i \epsilon)}, \zeta_{0}\right\rangle+\left\langle\overline{\tilde{u}_{0}}, \mathbf{Z}\left(\omega^{\prime}-i \epsilon^{\prime}\right)\right\rangle}{-(\omega+i \epsilon)+\omega^{\prime}-i \epsilon^{\prime}}\right],
\end{aligned}
$$

where we used (B5).

In the hyperfunction theory, the Dirac delta function $\delta(\omega)$ is defined by the well-known Plemelj formula;

$$
\frac{1}{\omega \pm i 0}=\mathrm{p} \cdot \mathrm{v} \cdot \frac{1}{\omega} \mp \pi i \delta(\omega), \quad \omega \in \mathbb{R}
$$

where p.v. denotes the principal value. Using this fact, the limit of $\epsilon^{\prime} \rightarrow+0$ followed by $\epsilon \rightarrow+0$ results in

$$
\left\langle\overline{\tilde{u}(\omega)}, \hat{\zeta}\left(\omega^{\prime}\right)\right\rangle=\frac{i}{2 \pi}\left[\frac{\left\langle\overline{\tilde{u}_{0}}, \hat{\zeta}\left(\omega^{\prime}\right)\right\rangle}{\omega^{\prime}-\omega+i 0}-\frac{\left\langle\overline{\tilde{u}_{0}}, \hat{\zeta}\left(\omega^{\prime}\right)\right\rangle}{\omega^{\prime}-\omega-i 0}\right]=\delta\left(\omega-\omega^{\prime}\right)\left\langle\overline{\tilde{u}_{0}}, \hat{\zeta}\left(\omega^{\prime}\right)\right\rangle .
$$

On the other hand, the limit of $\epsilon \rightarrow+0$ followed by $\epsilon^{\prime} \rightarrow+0$ gives

$$
\left\langle\overline{\hat{\tilde{u}}(\omega)}, \hat{\zeta}\left(\omega^{\prime}\right)\right\rangle=\frac{i}{2 \pi}\left[\frac{\left\langle\overline{\tilde{\tilde{u}}(\omega)}, \zeta_{0}\right\rangle}{\omega^{\prime}-\omega+i \epsilon^{\prime}}-\frac{\left\langle\overline{\hat{\tilde{u}}(\omega)}, \zeta_{0}\right\rangle}{\omega^{\prime}-\omega-i \epsilon^{\prime}}\right]=\delta\left(\omega-\omega^{\prime}\right)\left\langle\overline{\hat{\tilde{u}}(\omega)}, \zeta_{0}\right\rangle .
$$


Actually, we find that these two limits converge to the same value. To show this, let us substitute $\Omega=\overline{\Omega^{\prime}}$ into (B5), leaving

$$
\left\langle\overline{U(\bar{\Omega})}, \zeta_{0}\right\rangle+\left\langle\overline{\tilde{u}_{0}}, \mathrm{Z}(\Omega)\right\rangle=0
$$

Using this relation, we can confirm that

$$
\begin{aligned}
\left\langle\overline{\tilde{u}_{0}}, \hat{\zeta}(\omega)\right\rangle & =\frac{1}{2 \pi}\left[\left\langle\overline{\tilde{u}_{0}}, \mathbf{Z}(\omega+i 0)\right\rangle-\left\langle\overline{\tilde{u}_{0}}, \mathbf{Z}(\omega-i 0)\right\rangle\right] \\
& =\frac{1}{2 \pi}\left[-\left\langle\overline{U(\omega-i 0)}, \zeta_{0}\right\rangle+\left\langle\overline{U(\omega+i 0)}, \zeta_{0}\right\rangle\right] \\
& =\left\langle\overline{\hat{\tilde{u}}(\omega)}, \zeta_{0}\right\rangle .
\end{aligned}
$$

\section{APPENDIX C: ELIMINATION AND TRANSFORMATION OF VARIABLES}

Here, we newly pose a general problem that extracts only mathematical aspect of the symmetric response equation (39), and see how the elimination and transformation of variables work in this settings.

For that purpose, we consider a complex Hilbert space $L^{2}$ with an inner product $\langle\overline{\mathrm{o}}, \mathrm{o}\rangle$ and the following equation for $\mathbf{Z} \in L^{2}$,

$$
\mathcal{E}(\Omega) Z(\Omega)=-\chi(\Omega)
$$

where a linear operator $\mathcal{E}(\Omega): L^{2} \rightarrow L^{2}$ and $\chi(\Omega) \in L^{2}$ are given and depend on a parameter $\Omega \in \mathbb{C}$. Only the assumption is that the adjoint operator $\mathcal{E}^{*}(\Omega)$ satisfies $\mathcal{E}^{*}(\Omega)=\mathcal{E}(\bar{\Omega})$. The solution $\mathrm{Z}(\Omega)$ therefore depends on $\Omega$, too, and may not exist for some values of $\Omega$ at which $\mathcal{E}^{-1}(\Omega)$ is singular.

Define a function $D: \mathbb{C} \times L^{2} \times L^{2} \rightarrow \mathbb{C}$ by $D\left(\Omega, \mathrm{Z}_{1}, \mathrm{Z}_{2}\right):=\left\langle\mathrm{Z}_{1}, \mathcal{E}(\Omega) \mathrm{Z}_{2}\right\rangle$. Then, we obtain the following formula which turns out to be important in the main text.

\section{Proposition 10.}

$$
\begin{aligned}
-D(\Omega, \mathrm{Z}(\bar{\Omega}), \mathrm{Z}(\Omega))= & \langle\overline{\mathrm{Z}(\bar{\Omega})}, \chi(\Omega)\rangle=\langle\overline{\chi(\bar{\Omega})}, \mathrm{Z}(\Omega)\rangle, \\
= & \langle\overline{F(\Omega)}, \mathcal{E}(\Omega) F(\Omega)\rangle+\langle\overline{G(\Omega)}, \mathcal{E}(\Omega) G(\Omega)\rangle \\
& +\langle\overline{G(\Omega)}, \chi(\Omega)\rangle+\langle\overline{\chi(\bar{\Omega})}, G(\Omega)\rangle,
\end{aligned}
$$

where

$$
\begin{aligned}
F(\Omega) & =\frac{1}{2}[\mathrm{Z}(\Omega)-\mathrm{Z}(\bar{\Omega})], \\
G(\Omega) & =\frac{1}{2}[\mathrm{Z}(\Omega)+\mathrm{Z}(\bar{\Omega})] .
\end{aligned}
$$

Proof. The equality (C2) is trivial. To derive the last expression (C3), we begin with the following identity,

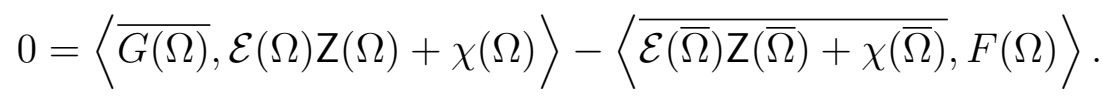


Substituting the relations $\mathrm{Z}(\Omega)=G(\Omega)+F(\Omega)$ and $\mathrm{Z}(\bar{\Omega})=G(\Omega)-F(\Omega)$, and using the properties $F(\Omega)=-F(\bar{\Omega})$ and $G(\Omega)=G(\bar{\Omega})$, this identity transforms into

$$
0=\langle\overline{G(\Omega)}, \mathcal{E}(\Omega) G(\Omega)\rangle+\langle\overline{G(\Omega)}, \chi(\Omega)\rangle+\langle\overline{F(\Omega)}, \mathcal{E}(\Omega) F(\Omega)\rangle-\langle\overline{\chi(\bar{\Omega})}, F(\Omega)\rangle .
$$

By substituting $F(\Omega)=\mathrm{Z}(\Omega)-G(\Omega)$ into the last term of the right hand side, the same expression as (C3) is obtained.

Now, suppose that there is an orthogonal decomposition $L^{2}=L_{a}^{2} \oplus L_{b}^{2}$ with inner products $\langle\bar{o}, \circ\rangle_{a}$ and $\langle\bar{o}, \circ\rangle_{b}$ for each subspaces. If we denote the corresponding decomposition by $\mathrm{Z}=\mathrm{Z}_{a}+\mathrm{Z}_{b}$, the equation can be written as

$$
\left(\begin{array}{ll}
\mathcal{E}_{a a}(\Omega) & \mathcal{E}_{a b}(\Omega) \\
\mathcal{E}_{b a}(\Omega) & \mathcal{E}_{b b}(\Omega)
\end{array}\right)\left(\begin{array}{l}
\mathrm{Z}_{a}(\Omega) \\
\mathrm{Z}_{b}(\Omega)
\end{array}\right)=-\left(\begin{array}{c}
\chi_{a}(\Omega) \\
\chi_{b}(\Omega)
\end{array}\right)
$$

We eliminate $\mathrm{Z}_{b}(\Omega)$ by prescribing the relation,

$$
\mathrm{Z}_{b}(\Omega)=-\mathcal{E}_{b b}^{-1}(\Omega) \mathcal{E}_{b a}(\Omega) \mathrm{Z}_{a}(\Omega)-\mathcal{E}_{b b}^{-1}(\Omega) \chi_{b}(\Omega)
$$

and obtain a reduced equation for $Z_{a}(\Omega) \in L_{a}^{2}$ as

$$
\left[\mathcal{E}_{a a}(\Omega)-\mathcal{E}_{a b}(\Omega) \mathcal{E}_{b b}^{-1}(\Omega) \mathcal{E}_{b a}(\Omega)\right] Z_{a}(\Omega)=-\chi_{a}(\Omega)+\mathcal{E}_{a b}(\Omega) \mathcal{E}_{b b}^{-1}(\Omega) \chi_{b}(\Omega)
$$

Furthermore, we introduce a variable transformation $Z_{a}(\Omega)=\mathcal{T}(\Omega) Z_{\sharp}(\Omega)$ by an operator $\mathcal{T}(\Omega): L_{a}^{2} \rightarrow L_{a}^{2}$ satisfying $\mathcal{T}^{*}(\Omega)=\mathcal{T}(\bar{\Omega})$. Then, we obtain a transformed equation

$$
\mathcal{E}_{\sharp}(\Omega) Z_{\sharp}(\Omega)=-\chi_{\sharp}(\Omega),
$$

where

$$
\begin{array}{r}
\mathcal{E}_{\sharp}(\Omega):=\mathcal{T}(\Omega)\left[\mathcal{E}_{a a}(\Omega)-\mathcal{E}_{a b}(\Omega) \mathcal{E}_{b b}^{-1}(\Omega) \mathcal{E}_{b a}(\Omega)\right] \mathcal{T}(\Omega), \\
\chi_{\sharp}(\Omega):=\mathcal{T}(\Omega) \chi_{a}(\Omega)-\mathcal{T}(\Omega) \mathcal{E}_{a b}(\Omega) \mathcal{E}_{b b}^{-1}(\Omega) \chi_{b}(\Omega) .
\end{array}
$$

The new operator $\mathcal{E}_{\sharp}(\Omega)$ satisfies $\mathcal{E}_{\sharp}^{*}(\Omega)=\mathcal{E}_{\sharp}(\bar{\Omega})$ again, and we therefore define a function $D_{\sharp}: \mathbb{C} \times L_{a}^{2} \times L_{a}^{2} \rightarrow \mathbb{C}$ also for this reduced equation (C10) by

$$
D_{\sharp}\left(\Omega, Z_{\sharp 1}, Z_{\sharp 2}\right):=\left\langle\overline{Z_{\sharp 1}}, \mathcal{E}_{\sharp}(\Omega) Z_{\sharp 2}\right\rangle_{a} .
$$

In this settings, the following formula holds;

\section{Proposition 11.}

$$
D(\Omega, \mathbf{Z}(\bar{\Omega}), \mathbf{Z}(\Omega))=D_{\sharp}\left(\Omega, \mathbf{Z}_{\sharp}(\bar{\Omega}), \mathrm{Z}_{\sharp}(\Omega)\right)+\left\langle\overline{\chi_{b}(\bar{\Omega})}, \mathcal{E}_{b b}^{-1}(\Omega) \chi_{b}(\Omega)\right\rangle_{b} .
$$

Proof. It is proven directly as follows.

$$
\begin{aligned}
& \left\langle\overline{Z_{\sharp}(\bar{\Omega})}, \chi_{\sharp}(\Omega)\right\rangle_{a}=\left\langle\overline{Z_{a}(\bar{\Omega})}, \chi_{a}(\Omega)-\mathcal{E}_{a b}(\Omega) \mathcal{E}_{b b}^{-1}(\Omega) \chi_{b}(\Omega)\right\rangle_{a}, \\
& =\left\langle\overline{Z_{a}(\bar{\Omega})}, \chi_{a}(\Omega)\right\rangle_{a}-\left\langle\overline{\mathcal{E}_{b b}^{-1}(\bar{\Omega}) \mathcal{E}_{b a}(\bar{\Omega}) Z_{a}(\bar{\Omega})}, \chi_{b}(\Omega)\right\rangle_{b}, \\
& =\left\langle\overline{Z_{a}(\bar{\Omega})}, \chi_{a}(\Omega)\right\rangle_{a}+\left\langle\overline{Z_{b}(\bar{\Omega})+\mathcal{E}_{b b}^{-1}(\bar{\Omega}) \chi_{b}(\bar{\Omega})}, \chi_{b}(\Omega)\right\rangle_{b}, \\
& =\langle\overline{\mathrm{Z}(\bar{\Omega})}, \chi(\Omega)\rangle+\left\langle\overline{\chi_{b}(\bar{\Omega})}, \mathcal{E}_{b b}^{-1}(\Omega) \chi_{b}(\Omega)\right\rangle_{b},
\end{aligned}
$$

where we used (C12) and (C8). 
Let $\sigma \subset \mathbb{C}$ be a set of $\Omega$ at which $D(\Omega, \mathbf{Z}(\bar{\Omega}), \mathrm{Z}(\Omega)$ ) becomes singular (i.e., poles, discontinuity and so on). Through the reduction of variables, we find that both the terms on the right hand side of (C14) would possibly contain more singularities other than $\sigma$, because the inverse operator $\mathcal{E}_{b b}^{-1}(\Omega)$ may not be defined or bounded for some parameters $\Omega$, say $\Omega \in \sigma_{b} \subset \mathbb{C}$. These new singularities $\sigma_{b}$ are clearly apparent. When applying the formula (C14) to the calculation of (41) and (42), there is no need to dwell on the apparent singularity $\sigma_{b}$ and one may naively replace $D(\Omega, \mathbf{Z}(\bar{\Omega}), \mathrm{Z}(\Omega))$ with $D_{\sharp}\left(\Omega, \mathrm{Z}_{\sharp}(\bar{\Omega}), \mathrm{Z}_{\sharp}(\Omega)\right)$ in $(41)$ and (42). However, in case that the genuine and apparent singularities degenerate $\left(\sigma \cap \sigma_{b} \neq \emptyset\right)$, the reduction does not work so effectively; one needs to retain the second term on the right hand side of (C14) for $\omega \in \sigma \cap \sigma_{b}$.

\section{ACKNOWLEDGMENTS}

The authors are grateful to Professor Zensho Yoshida for useful discussions and suggestions, and to Professor Eliezer Hameiri for stimulating our interest in this problem.

M. H. was supported by Research Fellowships of the Japan Society for the Promotion of Science (JSPS) for Young Scientists, and is supported by 21st century COE program "Development of Dynamic Mathematics with High Functionality" at Faculty of Mathematics, Kyushu University. Y. F. was supported in part by a Grant-in-Aid for scientific research from the Japan Society for the Promotion of Science.

[1] V. I. Arnold, Mathematical Methods of Classical Mechanics. Springer, Berlin (1978).

[2] V. I. Arnold and B. A. Khesin, Topological Methods in Hydrodynamics. Springer, New York (1998).

[3] D. D. Holm, J. E. Marsden, T. Ratiu and A. Weinstein, Phys. Rep. 123, 1 (1985).

[4] M. G. Krein, Dokl. Akad. Nauk SSSR A 73, 445 (1950).

[5] J. K. Moser, Comm. Pure Appl. Math. 11, 81 (1958).

[6] R. MacKay, Stability of equilibria of Hamiltonian systems, in Nonlinear Phenomena and Chaos. S. Sarkar (ed.), 254 (1986).

[7] P. L. Auer, H. Hurwitz and R. D. Miller, Phys. Fluids 1, 501 (1958).

[8] L. D. Landau and E. M. Lifshitz, Electrodynamics of continuous media. Addison-Wesley, Reading (1960).

[9] L. Brillouin, Wave Propagation and Group Velocity. Academic Press, New York (1960).

[10] T. H. Stix, The Theory of Plasma Waves. McGraw-Hill, New York (1962).

[11] P. J. Morrison and D. Pfirsch Phys. Fluids B, 4, 3038 (1992).

[12] N. G. Van Kampen, Physica 21, 949 (1955).

[13] R. A. Cairns, J. Fluid Mech., 92, 1 (1979).

[14] R. S. MacKay and P. G. Saffman, Proc. R. Soc. Lond. A 406, 115 (1986).

[15] N. J. Balmforth and P. Morrison, Hamiltonian description of shear flow. In Large-Scale Atmosphere-Ocean Dynamics 2: Geometric Methods and Models. Eds. J. Norbury and I. Roulstone, Cambridge University Press, 117 (2002).

[16] D. D. Holm, B. A. Kupershmidt and C. D. Levermore, Phys. Lett., 93A, 389 (1983).

[17] J. E. Marsden, T. Ratiu and A. Weinstein, Trans. Am. Math. Soc., 281, 147 (1984).

[18] J. E. Marsden and A. Weinstein, Physica D, 7, 305 (1983). 
[19] E. Frieman and M. Rotenberg, Rev. Mod. Phys. 32, 898 (1960).

[20] E. Hameiri, Phys. Plasmas 10, 2643 (2003).

[21] K. Hain and R. Lüst, Z. Naturforsch. 13a, 936 (1958).

[22] J. P. Goedbloed, Physica, 53, 412 (1971); Physica, 53, 501 (1971).

[23] F. P. Bretherton, Quart. J. Roy. Meteor. Soc. 92, 325 (1966).

[24] K. Iga, Fluid Dyn. Res. 25, 63 (1999).

[25] R. J. Briggs, J. D. Daugherty and R. H. Levy, Phys. Fluids 13, 421 (1970).

[26] P. J. Morrison, J. M. Greene, Phys. Rev. Lett., 45, 790 (1980).

[27] P. J. Morrison, Rev. Mod. Phys. 70, 467 (1998).

[28] S. Friedlander and M. M. Vishik, Geophys. Astrophys. Fluid Dynamics, 55, 19 (1990).

[29] M. B. Isichenko, Phys. Rev. Lett., 80, no. 5, 972 (1998).

[30] I. Imai, Applied hyperfunction theory, edited by M. Hazewinkel (Kluwer Academic Pub., Dordrecht, 1992).

[31] T. Kato, Perturbation Theory for Linear Operators (Springer-Verlag, New York, 1976).

[32] M. Hirota, T. Tatsuno, and Z. Yoshida, Phys. Plasmas 12, 012107 (2005).

[33] K. M. Case, Ann. Phys. 7, 349 (1959).

[34] K. M. Case, Phys. Fluids 3, 143 (1960).

[35] Z. Sedláček, J. Plasma Phys., 5, 239 (1971).

[36] J. Tataronis and W. Grossmann, Z. Physik, 261, 203 (1973).

[37] M. Hirota, T. Tatsuno, Z. Yoshida, J. Plasma Phys. 69, 397 (2003).

[38] H. K. Moffatt, Phil Trans. R. Soc. Lond. A 333, 321 (1990).

[39] I. B. Bernstein, E. A. Frieman, M. D. Kruskal and R. M. Kulsrud, Proc. Roy. Soc. London A244, 17 (1958).

[40] E. Hameiri, Phys. Plasmas 5, 3270 (1998).

[41] K. Appert, R. Gruber and J. Vaclavik, Phys. Fluids, 17, 1471 (1974).

[42] L. Lees and C. C. Lin, NACA Technical Note No. 1115 (1946).

[43] P. G. Drazin and W. H. Reid, Hydrodynamic stability, (Cambridge Univ., Cambridge, 1981).

[44] J. W. S. Rayleigh, Proc. Lond. Math. Soc. 9, 57 (1880).

[45] A. Eliassen, E. Høiland and E. Riis, Inst. for Weather and Climate Res., Pub. No. 1, Oslo (1953). 


\section{List of MHF Preprint Series, Kyushu University \\ 21st Century COE Program \\ Development of Dynamic Mathematics with High Functionality}

MHF2005-1 Hideki KOSAKI

Matrix trace inequalities related to uncertainty principle

MHF2005-2 Masahisa TABATA

Discrepancy between theory and real computation on the stability of some finite element schemes

MHF2005-3 Yuko ARAKI \& Sadanori KONISHI

Functional regression modeling via regularized basis expansions and model selection

MHF2005-4 Yuko ARAKI \& Sadanori KONISHI

Functional discriminant analysis via regularized basis expansions

MHF2005-5 Kenji KAJIWARA, Tetsu MASUDA, Masatoshi NOUMI, Yasuhiro OHTA \& Yasuhiko YAMADA

Point configurations, Cremona transformations and the elliptic difference Painlevé equations

MHF2005-6 Kenji KAJIWARA, Tetsu MASUDA, Masatoshi NOUMI, Yasuhiro OHTA \& Yasuhiko YAMADA

Construction of hypergeometric solutions to the $q$ - Painlevé equations

MHF2005-7 Hiroki MASUDA

Simple estimators for non-linear Markovian trend from sampled data:

I. ergodic cases

MHF2005-8 Hiroki MASUDA \& Nakahiro YOSHIDA

Edgeworth expansion for a class of Ornstein-Uhlenbeck-based models

MHF2005-9 Masayuki UCHIDA

Approximate martingale estimating functions under small perturbations of dynamical systems

MHF2005-10 Ryo MATSUZAKI \& Masayuki UCHIDA

One-step estimators for diffusion processes with small dispersion parameters from discrete observations

MHF2005-11 Junichi MATSUKUBO, Ryo MATSUZAKI \& Masayuki UCHIDA

Estimation for a discretely observed small diffusion process with a linear drift

MHF2005-12 Masayuki UCHIDA \& Nakahiro YOSHIDA

AIC for ergodic diffusion processes from discrete observations 
MHF2005-13 Hiromichi GOTO \& Kenji KAJIWARA

Generating function related to the Okamoto polynomials for the Painlevé IV equation

MHF2005-14 Masato KIMURA \& Shin-ichi NAGATA

Precise asymptotic behaviour of the first eigenvalue of Sturm-Liouville problems with large drift

MHF2005-15 Daisuke TAGAMI \& Masahisa TABATA

Numerical computations of a melting glass convection in the furnace

MHF2005-16 Raimundas VIDŪNAS

Normalized Leonard pairs and Askey-Wilson relations

MHF2005-17 Raimundas VIDŪNAS

Askey-Wilson relations and Leonard pairs

MHF2005-18 Kenji KAJIWARA \& Atsushi MUKAIHIRA

Soliton solutions for the non-autonomous discrete-time Toda lattice equation

MHF2005-19 Yuu HARIYA

Construction of Gibbs measures for 1-dimensional continuum fields

MHF2005-20 Yuu HARIYA

Integration by parts formulae for the Wiener measure restricted to subsets in $\mathbb{R}^{d}$

MHF2005-21 Yuu HARIYA

A time-change approach to Kotani's extension of Yor's formula

MHF2005-22 Tadahisa FUNAKI, Yuu HARIYA \& Mark YOR

Wiener integrals for centered powers of Bessel processes, I

MHF2005-23 Masahisa TABATA \& Satoshi KAIZU

Finite element schemes for two-fluids flow problems

MHF2005-24 Ken-ichi MARUNO \& Yasuhiro OHTA

Determinant form of dark soliton solutions of the discrete nonlinear Schrödinger equation

MHF2005-25 Alexander V. KITAEV \& Raimundas VIDŪNAS

Quadratic transformations of the sixth Painlevé equation

MHF2005-26 Toru FUJII \& Sadanori KONISHI

Nonlinear regression modeling via regularized wavelets and smoothing parameter selection

MHF2005-27 Shuichi INOKUCHI, Kazumasa HONDA, Hyen Yeal LEE, Tatsuro SATO, Yoshihiro MIZOGUCHI \& Yasuo KAWAHARA

On reversible cellular automata with finite cell array 
MHF2005-28 Toru KOMATSU

Cyclic cubic field with explicit Artin symbols

MHF2005-29 Mitsuhiro T. NAKAO, Kouji HASHIMOTO \& Kaori NAGATOU

A computational approach to constructive a priori and a posteriori error estimates for finite element approximations of bi-harmonic problems

MHF2005-30 Kaori NAGATOU, Kouji HASHIMOTO \& Mitsuhiro T. NAKAO

Numerical verification of stationary solutions for Navier-Stokes problems

MHF2005-31 Hidefumi KAWASAKI

A duality theorem for a three-phase partition problem

MHF2005-32 Hidefumi KAWASAKI

A duality theorem based on triangles separating three convex sets

MHF2005-33 Takeaki FUCHIKAMI \& Hidefumi KAWASAKI

An explicit formula of the Shapley value for a cooperative game induced from the conjugate point

MHF2005-34 Hideki MURAKAWA

A regularization of a reaction-diffusion system approximation to the two-phase

Stefan problem

MHF2006-1 Masahisa TABATA

Numerical simulation of Rayleigh-Taylor problems by an energy-stable finite element scheme

MHF2006-2 Ken-ichi MARUNO \& G R W QUISPEL

Construction of integrals of higher-order mappings

MHF2006-3 Setsuo TANIGUCHI

On the Jacobi field approach to stochastic oscillatory integrals with quadratic phase function

MHF2006-4 Kouji HASHIMOTO, Kaori NAGATOU \& Mitsuhiro T. NAKAO

A computational approach to constructive a priori error estimate for finite element approximations of bi-harmonic problems in nonconvex polygonal domains

MHF2006-5 Hidefumi KAWASAKI

A duality theory based on triangular cylinders separating three convex sets in $R^{n}$

MHF2006-6 Raimundas VIDŪNAS

Uniform convergence of hypergeometric series

MHF2006-7 Yuji KODAMA \& Ken-ichi MARUNO

N-Soliton solutions to the DKP equation and Weyl group actions 
MHF2006-8 Toru KOMATSU

Potentially generic polynomial

MHF2006-9 Toru KOMATSU

Generic sextic polynomial related to the subfield problem of a cubic polynomial

MHF2006-10 Shu TEZUKA \& Anargyros PAPAGEORGIOU

Exact cubature for a class of functions of maximum effective dimension

MHF2006-11 Shu TEZUKA

On high-discrepancy sequences

MHF2006-12 Raimundas VIDŪNAS

Detecting persistent regimes in the North Atlantic Oscillation time series

MHF2006-13 Toru KOMATSU

Tamely Eisenstein field with prime power discriminant

MHF2006-14 Nalini JOSHI, Kenji KAJIWARA \& Marta MAZZOCCO

Generating function associated with the Hankel determinant formula for the solutions of the Painlevé IV equation

MHF2006-15 Raimundas VIDŪNAS

Darboux evaluations of algebraic Gauss hypergeometric functions

MHF2006-16 Masato KIMURA \& Isao WAKANO

New mathematical approach to the energy release rate in crack extension

MHF2006-17 Toru KOMATSU

Arithmetic of the splitting field of Alexander polynomial

MHF2006-18 Hiroki MASUDA

Likelihood estimation of stable Lévy processes from discrete data

MHF2006-19 Hiroshi KAWABI \& Michael RÖCKNER

Essential self-adjointness of Dirichlet operators on a path space with Gibbs measures via an SPDE approach

MHF2006-20 Masahisa TABATA

Energy stable finite element schemes and their applications to two-fluid flow problems

MHF2006-21 Yuzuru INAHAMA \& Hiroshi KAWABI

Asymptotic expansions for the Laplace approximations for Itô functionals of Brownian rough paths

MHF2006-22 Yoshiyuki KAGEI

Resolvent estimates for the linearized compressible Navier-Stokes equation in an infinite layer 
MHF2006-23 Yoshiyuki KAGEI

Asymptotic behavior of the semigroup associated with the linearized compressible Navier-Stokes equation in an infinite layer

MHF2006-24 Akihiro MIKODA, Shuichi INOKUCHI, Yoshihiro MIZOGUCHI \& Mitsuhiko FUJIO

The number of orbits of box-ball systems

MHF2006-25 Toru FUJII \& Sadanori KONISHI

Multi-class logistic discrimination via wavelet-based functionalization and model selection criteria

MHF2006-26 Taro HAMAMOTO, Kenji KAJIWARA \& Nicholas S. WITTE

Hypergeometric solutions to the $q$-Painlevé equation of type $\left(A_{1}+A_{1}^{\prime}\right)^{(1)}$

MHF2006-27 Hiroshi KAWABI \& Tomohiro MIYOKAWA

The Littlewood-Paley-Stein inequality for diffusion processes on general metric spaces

MHF2006-28 Hiroki MASUDA

Notes on estimating inverse-Gaussian and gamma subordinators under highfrequency sampling

MHF2006-29 Setsuo TANIGUCHI

The heat semigroup and kernel associated with certain non-commutative harmonic oscillators

MHF2006-30 Setsuo TANIGUCHI

Stochastic analysis and the KdV equation

MHF2006-31 Masato KIMURA, Hideki KOMURA, Masayasu MIMURA, Hidenori MIYOSHI, Takeshi TAKAISHI \& Daishin UEYAMA

Quantitative study of adaptive mesh FEM with localization index of pattern

MHF2007-1 Taro HAMAMOTO \& Kenji KAJIWARA

Hypergeometric solutions to the $q$-Painlevé equation of type $A_{4}^{(1)}$

MHF2007-2 Kouji HASHIMOTO, Kenta KOBAYASHI \& Mitsuhiro T. NAKAO

Verified numerical computation of solutions for the stationary Navier-Stokes equation in nonconvex polygonal domains

MHF2007-3 Kenji KAJIWARA, Marta MAZZOCCO \& Yasuhiro OHTA

A remark on the Hankel determinant formula for solutions of the Toda equation

MHF2007-4 Jun-ichi SATO \& Hidefumi KAWASAKI

Discrete fixed point theorems and their application to Nash equilibrium

MHF2007-5 Mitsuhiro T. NAKAO \& Kouji HASHIMOTO

Constructive error estimates of finite element approximations for non-coercive elliptic problems and its applications 
MHF2007-6 Kouji HASHIMOTO

A preconditioned method for saddle point problems

MHF2007-7 Christopher MALON, Seiichi UCHIDA \& Masakazu SUZUKI

Mathematical symbol recognition with support vector machines

MHF2007-8 Kenta KOBAYASHI

On the global uniqueness of Stokes' wave of extreme form

MHF2007-9 Kenta KOBAYASHI

A constructive a priori error estimation for finite element discretizations in a non-convex domain using singular functions

MHF2007-10 Myoungnyoun KIM, Mitsuhiro T. NAKAO, Yoshitaka WATANABE \& Takaaki NISHIDA

A numerical verification method of bifurcating solutions for 3-dimensional Rayleigh-Bénard problems

MHF2007-11 Yoshiyuki KAGEI

Large time behavior of solutions to the compressible Navier-Stokes equation in an infinite layer

MHF2007-12 Takashi YANAGAWA, Satoshi AOKI and Tetsuji OHYAMA

Human finger vein images are diverse and its patterns are useful for personal identification

MHF2007-13 Masahisa TABATA

Finite element schemes based on energy-stable approximation for two-fluid flow problems with surface tension

MHF2007-14 Mitsuhiro T. NAKAO \& Takehiko KINOSHITA

Some remarks on the behaviour of the finite element solution in nonsmooth domains

MHF2007-15 Yoshiyuki KAGEI \& Takumi NUKUMIZU

Asymptotic behavior of solutions to the compressible Navier-Stokes equation in a cylindrical domain

MHF2007-16 Shuichi INOKUCHI, Yoshihiro MIZOGUCHI, Hyen Yeal LEE \& Yasuo KAWAHARA

Periodic Behaviors of Quantum Cellular Automata

MHF2007-17 Makoto HIROTA \& Yasuhide FUKUMOTO

Energy of hydrodynamic and magnetohydrodynamic waves with point and continuous spectra 